\title{
SPACE AND ENERGY EFFECTS IN REACTOR FLUCTUATION EXPERIMENTS
}

\author{
M. Nateison, * R. K. Osborn and F. Shure \\ Department of Nuclear Engineering, The University of Michigan, Ann Arbor, Michigan
}

(Received 27 September 1965)

\begin{abstract}
An analysis of the space and energy dependence of neutron fluctuations is presented, and applied to the results of reactor fluctuation experiments. Two experiments, measurements of Power Spectral Density and Variance to Mean, are singled out for special attention. These are of special interest, since previously published experimental results seem to be interpretable by space and energyindependent theories of neutron fluctuations.

Both infinite and finite-geometry reactor models are treated, and calculations are presented to illustrate the effects of detector size, shape and location, and delayed neutrons. In any event, agreement with actual experimental results is achieved only when the effect of fast diffusion is included through a two-energy group model. It is seen that when the migration length is sufficiently large by comparison with a typical reactor dimension, the results of the space-energy independent theory appear as a first approximation. Thus it is argued that experiments on larger reactors would yield results not in agreement with the space-energy independent theory.
\end{abstract}

\section{INTRODUCTION}

OUR purpose in this paper will be to attempt a careful analysis of two types of experiments which measure neutron fluctuations in a steady-state reactor. In the first type, the power spectral density (P.S.D.) of the current produced in a single detector in or near the reactor is measured. In the second, the ratio of the variance-to-mean $(V / M)$ of the number of counts recorded by a detector as a function of the counting interval is measured. The first is typified by a P.S.D. measurement performed by RICKER et al. (1965) on the ORNL pool-type critical facility, and the second by a $V / M$ measurement by ALBRECHT (1962) on the University of Michigan Ford Nuclear Reactor. Motivation for the consideration of these experiments, and for the related studies discussed here, stems from the desire to understand the success of a seemingly space and energy-independent theory of neutron fluctuations (RICKER et al; AlBRECHT) in interpreting the data. Of course, experiments which measure the kinetics of the mean neutron distribution can often be interpreted by a space and energyindependent theory (i.e. point reactor kinetics) in which fast and thermal leakage rates are included. This is accomplished by assuming a space-time decoupling of the mean neutron distribution in a finite reactor. Thus, the absorption rate in the point kinetic equations is modified to include the fast and thermal leakage rates. But there is no reason to believe a similar decoupling can be applicd to the higher moments of the neutron distribution which describe neutron fluctuations. It will be part of the burden of this paper to show that in general there is no similar decoupling for the higher moments, but that for the specific systems considered by RICKER and ALBRECHT an approximate decoupling does occur which makes possible the interpretation of experiments performed on them by a space and energy-independent theory, a theory in which infinite reactor parameters are replaced by appropriate finite reactor parameters.

* A.E.C. pre-doctoral fellow. 
Our programme is as follows:

(a) First we explore the relations between the quantities measured in P.S.D. and $V / M$ experiments and the various moments of the neutron distribution.

(b) We then evaluate the effects on the results of these experiments due to

(i) Detector Volume (SHEFF, 1965)

(ii) Detector location with respect to the reactor (SHEFF)

(iii) Detector geometry

(iv) Reactor Volume (SHEFF; HARRIS, 1965)

(v) Delayed neutron production, (ShefF; RAiEvski, 1960; OsborN and AKCASU, 1965).*

(c) Finally we apply the above to the specific experiments of RICKER et al. and ALBRECHT, and propose certain new experiments.

The entire analysis will be performed in the framework of a physical, rather than phenomenological, theory of neutron fluctuations. The theory has been introduced previously by the authors (OSBORN and YIP, 1963; OSBORN and NATELSON, 1965) and detailed derivations are presented there.

In the physical theory the basic quantities are: $N^{D}(\mathbf{x}, t)$, the detected particle singlet density, the expected number of detected particles (e.g. $\alpha$-particles in a $\mathrm{BF}_{3}$ detector) accumulated per unit volume about $\mathbf{x}$ in a counting interval of $t \mathrm{sec}$; and $N^{D D}\left(\mathbf{x}, \mathbf{x}^{\prime}, t\right)$ the detected particle doublet density, the expected product of the number of detected particles accumulated per unit volume about $\mathbf{x}$ and the number accumulated per unit volume about $\mathbf{x}^{\prime}$ in a counting interval of $t$ sec. They are related to the first and second moments of the neutron distribution-the neutron singlet and doublet densities, $N^{N}(\mathbf{x}, \mathbf{v}, t)$ and $N^{N N}\left(\mathbf{x}, \mathbf{v}, \mathbf{x}^{\prime}, \mathbf{v}^{\prime}, t\right)$ and thus to the various reactor parameters.

In a P.S.D. experiment the frequency spectrum of the fluctuations in the current from an ionization chamber in or near the reactor is measured. This spectrum $\Phi(\omega)$ is obtained experimentally from the cosine transform of the chamber current autocovariance, i.e.

where

$$
\Phi(\omega)=\int_{0}^{\infty} \mathrm{d} t \cos \omega t \phi(t)
$$

$$
\phi(t)=\lim _{T \rightarrow \infty} \frac{1}{T} \int_{0}^{T} \mathrm{~d} r i(r) i(r+t)
$$

is the chamber current autocovariance, $\dagger$ and $i(r)=\mathscr{I}(r)-I$ is the fluctuation of the chamber current; $\mathscr{I}(r)$ being the instantaneous chamber current and $I$ the steady-state or d.c. chamber current. $\neq$ The autocovariance, $\phi(t)$, can be related directly to the detected particle correlation function, $\mathrm{X}^{D D}\left(\mathbf{x}, \mathbf{x}^{\prime}, t\right)$, according to

$$
\phi(t)=\frac{\Gamma^{2}}{2} \int_{\text {D.V. }} \mathrm{d}^{3} \mathbf{x} \int_{\text {D.v. }} \mathrm{d}^{3} \mathbf{x} \frac{\partial^{2}}{\partial t^{2}} \mathrm{X}^{D D}\left(\mathbf{x}, \mathbf{x}^{\prime}, t\right),
$$

* The role of delayed neutrons in noise analysis experiments has received such extensive attention, that we have referenced only three papers, each representing a different approach.

$\dagger$ Of course a given experiment measures only an approximate value for $\phi(t)$.

$\ddagger$ Whether $\phi(t)$ is measured with respect to $i(t)$ or $\mathscr{I}(t)$ is of no consequence to the P.S.D. measurement, as the difference would only appear at zero frequency. 
where $\mathrm{X}^{D D}\left(\mathbf{x}, \mathbf{x}^{\prime}, t\right)=N^{D D}\left(\mathbf{x}, \mathbf{x}^{\prime}, t\right)-N^{D}(\mathbf{x}, t) N^{D}\left(\mathbf{x}^{\prime}, t\right)$, D.V. = detector volume, and $\Gamma$ is an appropriate conversion factor from detection rate to current. A derivation of this relationship has been presented in the spirit of the physical theory by one of us (OsBorn, 1965) and by others using various inductive arguments (Pluta, 1961; SHeFF and Albrecht, 1964; BenNeTt, 1960). Entering (2) into (1) yields the desired relation between the measured P.S.D. and the detected particle correlation function of the physical theory, i.e.

$$
\Phi(\omega)=\frac{\Gamma^{2}}{2} \int_{0}^{\infty} \mathrm{d} t \cos \omega t \int_{\mathrm{n} . \mathrm{V} .} \mathrm{d}^{3} \mathbf{x} \int_{\text {D.v. }} \mathrm{d}^{3} \mathbf{x}^{\prime} \frac{\partial^{2}}{\partial t^{2}} \mathrm{X}^{D D}\left(\mathbf{x}, \mathbf{x}^{\prime}, t\right) .
$$

In a $V / M$ experiment a time record is made of the current pulses produced in an ionization chamber located in or near a reactor. The record is divided into equal intervals and the mean $\bar{n}$ and mean square $\overline{n^{2}}$ number of pulses recorded for a given interval are computed. This process is repeated for intervals of different duration. Thus the variance-to-mean, $\left(\overline{n^{2}}-\bar{n}^{2}\right) /, \bar{n}$ as a function of counting interval, can be computed. By definition, the relationship of the detected particle densities to this measured quantity is

$$
V / M(r)=\frac{\overline{n^{2}}-\bar{n}^{2}}{\bar{n}}(r)=\int_{\text {D.V. }} \mathrm{d}^{3} \mathbf{x} \int_{\text {D.V. }} \mathrm{d}^{3} \mathbf{x}^{\prime} \mathbf{X}^{D D}\left(\mathbf{x}, \mathbf{x}^{\prime}, r\right) / \int_{\text {D.F. }} \mathrm{d}^{3} \mathbf{x} N^{D}(\mathbf{x}, r)
$$

It is clear from relations (3) and (4) that the P.S.D. and $V / M$ experiments are closely related; both in some sense measure $\mathrm{X}^{D D}\left(\mathbf{x}, \mathbf{x}^{\prime}, t\right)$, the detected particle correlation function. Of course the technical problems in handling currents and counting records are quite different. Thus, the limits of applicability for the two experiments will not be the same. However, for the reactors (small cold clean cores) and power levels (milliwatts) involved in the experiments of Albrecht, and Ricker et al. either experiment could have been done on either system. We will, therefore, be able to consider simultaneously effects on both experiments of the various reactordetector properties.

In Section 2 the set of equations relating the detected particle and neutron densities is introduced. A reduction from the transport to a diffusion description is made in a mathematically consistent manner. The resulting set of working equations is then applied to the second and third phases of the analysis in the subsequent sections.

In Section 3 an infinite-volume model of a thermal reactor is considered, and the effect of detector configuration is investigated for P.S.D. and $V / M$ experiments which might be performed on a reactor described by such a model. The results are not in accordance with the experiments of either Ricker or Albrecht. In fact, the experimental measurements agree with computations using an infinite detector, whereas if detector geometry similar to the actual detectors is used in the calculation, the results are substantially different from the measurements.

In Section 4, we study a finite, cubical reactor. A particular reactor-detector configuration is considered which is intended to represent the systems studied by both Ricker and Albrecht. Here again, if we assume as before that the neutron population is adequately described by considering only thermal diffusion, the results are not in agreement with these experiments.

In Section 5 we incorporate diffusion during moderation into the reactor model of Section 6. We obtain immediate agreement with the experimental results. We note that for reactors of the type utilized by both Ricker and Albrecht this modification 
effectively eliminates higher mode contributions to the spatial dependence of the various correlation functions and thereby we are led to an explanation (and also a qualification) of the success of the space-energy independent theory.

In this section we also consider hypothetical P.S.D. experiments on a large graphitemoderated reactor with various detector configurations and positions. The results obtained demonstrate the need to include the effect of finite reactor size, even for a reactor of this magnitude. The infinite reactor model of Section 3 will be shown to have only limited applicability for such a reactor.

\section{THE BASIC EQUATIONS}

The system of equations relating the measured quantities, $N^{D D}\left(\mathbf{x}, \mathbf{x}^{\prime}, t\right)$ and $N^{D}(\mathbf{x}, t)$, to the first and second moments of the neutron distribution and thus to the various reactor parameters have been derived previously (Osborn and Yip; Osborn and Natelson). This derivation will not be reproduced here, though it is well to recall two of the approximations involved: the neglect of neutron-neutron collisions, and the neglect of correlations between the neutron and reactor nuclei distributions. For brevity the equations will be displayed in reduced form. Delayed neutrons are ignored, and averages are taken over particle speeds. The energyaveraged parameters (e.g. scattering and absorption rates) are assumed equal, whether a singlet or a doublet density has acted as a weight function in the averaging process. This assumption is implicit in all theories of neutron noise where energy dependence is not explicitly considered. The delayed neutrons, on the other hand, have been ignored only for simplicity. Their effect on measurements in the time and frequency intervals of interest here is considered in Appendix A and seen to be negligible. Our working set of equations (with the above considerations in mind) are exhibited as equations (5)-(8), (10), (11):

$$
\begin{aligned}
& \frac{\partial N^{D}(\mathbf{x}, t)}{\partial t}=r_{D}(\mathbf{x}) \int \mathrm{d} \boldsymbol{\Omega} N^{N}(\mathbf{x}, \boldsymbol{\Omega}, t) . \\
& \frac{\partial}{\partial t} N^{D D}\left(\mathbf{x}, \mathbf{x}^{\prime}, t\right)=r_{D}(\mathbf{x}) \int \mathrm{d} \boldsymbol{\Omega} N^{N D}\left(\mathbf{x}, \boldsymbol{\Omega}, \mathbf{x}^{\prime}, t\right)+r_{D}\left(\mathbf{x}^{\prime}\right) \int \mathrm{d} \boldsymbol{\Omega}^{\prime} N^{N D}\left(\mathbf{x}^{\prime}, \boldsymbol{\Omega}^{\prime} \mathbf{x}, t\right) \\
& +r_{D}(\mathbf{x}) \delta\left(\mathbf{x}-\mathbf{x}^{\prime}\right) \int \mathrm{d} \boldsymbol{\Omega} N^{N}(\mathbf{x}, \boldsymbol{\Omega}, t) . \\
& {\left[\frac{\partial}{\partial t}+B\right] N^{N D}\left(\mathbf{x}, \boldsymbol{\Omega}, \mathbf{x}^{\prime}, t\right)=r_{D}\left(\mathbf{x}^{\prime}\right) \int \mathrm{d} \boldsymbol{\Omega}^{\prime} N^{N N}\left(\mathbf{x}, \boldsymbol{\Omega}, \mathbf{x}^{\prime}, \mathbf{\Omega}^{\prime}, t\right)+\frac{S(\mathbf{x}, t)}{4 \pi} N^{D}(\mathbf{x}, t)} \\
& -r_{D}\left(\mathbf{x}^{\prime}\right) \delta\left(\mathbf{x}-\mathbf{x}^{\prime}\right) N^{N}(\mathbf{x}, \boldsymbol{\Omega}, t) \text {. } \\
& {\left[\frac{\partial}{\partial t}+B\right] N^{N D}\left(\mathbf{x}^{\prime}, \boldsymbol{\Omega}^{\prime}, \mathbf{x}, t\right)=r_{D}(\mathbf{x}) \int \mathrm{d} \boldsymbol{\Omega} N^{N N}\left(\mathbf{x}, \boldsymbol{\Omega}, \mathbf{x}^{\prime}, \boldsymbol{\Omega}^{\prime}, t\right)+\frac{S\left(\mathbf{x}^{\prime}, t\right)}{4 \pi} N^{D}(\mathbf{x}, t)} \\
& -r_{D}(\mathbf{x}) \delta\left(\mathbf{x}-\mathbf{x}^{\prime}\right) N^{N}\left(\mathbf{x}^{\prime}, \mathbf{\Omega}^{\prime}, t\right)
\end{aligned}
$$

where $S(\mathbf{x}, t) / 4 \pi$ is the expected number of neutrons produced by external sources, per unit volume about $\mathbf{x}$, per second (production assumed isotropic in velocity) and where the operator $B$ is defined according to

$$
\begin{gathered}
B N(\mathbf{x}, \boldsymbol{\Omega})=\langle v\rangle \boldsymbol{\Omega} \cdot \boldsymbol{\nabla} N(\mathbf{x}, \boldsymbol{\Omega})+r_{t} N(\mathbf{x}, \boldsymbol{\Omega}) \\
-r_{s} \int \mathrm{d} \boldsymbol{\Omega}^{\prime \prime} F\left(\boldsymbol{\Omega}^{\prime \prime} \rightarrow \boldsymbol{\Omega}\right) N\left(\mathbf{x}, \boldsymbol{\Omega}^{\prime \prime}\right)-\frac{\langle j\rangle}{4 \pi} r_{f} \int \mathrm{d} \boldsymbol{\Omega}^{\prime \prime} N\left(\mathbf{x}, \boldsymbol{\Omega}^{\prime \prime}\right) .
\end{gathered}
$$

The conditional probability that a neutron having been scattered while travelling in 
direction $\boldsymbol{\Omega}^{\prime}$, will be found travelling in the solid angle $\mathrm{d} \boldsymbol{\Omega}$ about $\boldsymbol{\Omega}$ is represented by $F\left(\boldsymbol{\Omega}^{\prime} \rightarrow \boldsymbol{\Omega}\right) \mathrm{d} \boldsymbol{\Omega}$, the scattering frequency.

$$
\begin{aligned}
& {\left[\frac{\partial}{\partial t}+B\right] N^{N^{\prime}}(\mathbf{x}, \Omega, t)=S(\mathbf{x}, t) / 4 \pi .} \\
& {\left[\frac{\partial}{\partial t}+B+B^{\prime}\right] N^{N y}\left(\mathbf{x}, \boldsymbol{\Omega}, \mathbf{x}^{\prime}, \boldsymbol{\Omega}^{\prime}, t\right)=\frac{S(\mathbf{x}, t)}{4 \pi} N^{-Y}\left(\mathbf{x}^{\prime}, \boldsymbol{\Omega}^{\prime}, t\right)+\frac{S\left(\mathbf{x}^{\prime}, t\right)}{4 \pi} N^{N}(\mathbf{x}, \boldsymbol{\Omega}, t)} \\
& +\delta\left(\mathbf{x}-\mathbf{x}^{\prime}\right) \delta\left(\boldsymbol{\Omega}-\boldsymbol{\Omega}^{\prime}\right) S(\mathbf{x}, t) / 4 \pi \\
& +\delta\left(\mathbf{x}-\mathbf{x}^{\prime}\right) \Gamma N^{\prime \prime}(\mathbf{x}, \boldsymbol{\Omega}, t),
\end{aligned}
$$

where

$$
\begin{aligned}
\Gamma N^{N}(\mathbf{x}, \boldsymbol{\Omega}, t)= & \delta\left(\boldsymbol{\Omega}-\boldsymbol{\Omega}^{\prime}\right)\left\{r_{t} N^{N}(\mathbf{x}, \boldsymbol{\Omega}, t)+r_{s} \int \mathrm{d} \boldsymbol{\Omega}^{\prime \prime} F\left(\boldsymbol{\Omega}^{\prime \prime} \rightarrow \boldsymbol{\Omega}\right) N^{v}\left(\mathbf{x}, \boldsymbol{\Omega}^{\prime \prime}, t\right)\right. \\
& \left.+\frac{\left\langle j^{2}\right\rangle}{4 \pi} r_{f} \int \mathrm{d} \boldsymbol{\Omega}^{\prime \prime} N^{N}\left(\mathbf{x}, \boldsymbol{\Omega}^{\prime \prime}, t\right)\right\}-r_{s} F\left(\boldsymbol{\Omega}^{\prime} \rightarrow \boldsymbol{\Omega}\right) N^{N}\left(\mathbf{x}, \boldsymbol{\Omega}^{\prime}, t\right) \\
& -r_{s} F\left(\boldsymbol{\Omega} \rightarrow \boldsymbol{\Omega}^{\prime}\right) N^{N}(\mathbf{x}, \boldsymbol{\Omega}, t)-\frac{j j\rangle}{4 \pi} r_{f}\left\{N^{N}(\mathbf{x}, \boldsymbol{\Omega}, t)+N^{x}\left(\mathbf{x}, \boldsymbol{\Omega}^{\prime}, t\right)\right\} .
\end{aligned}
$$

The various parameters appearing above are defined as follows: The ' $r$ 's refer to the reaction rates, $r_{t}$ for total, $r_{s}$ for scattering, $r_{f}$ for fission and $r_{D}$ for detection. $\langle v\rangle$ is the mean neutron speed, $\langle j\rangle$ the mean number of prompt neutrons produced per fission, and $\left\langle j^{2}\right\rangle$ the mean square number per fission. The production of neutrons by fission is assumed to be isotropic.

The equations are reduced by a truncated spherical harmonics expansion.

For densities which depend on a single-direction vector we have:

$$
N(\mathbf{x}, \boldsymbol{\Omega})=\frac{N(\mathbf{x})}{4 \pi}+\frac{3}{4 \pi} \boldsymbol{\Omega} \cdot \boldsymbol{\phi}(\mathbf{x}) ;
$$

and for those dependent on two direction vectors,

$$
\begin{aligned}
N\left(\mathbf{x}, \boldsymbol{\Omega}, \mathbf{x}^{\prime}, \boldsymbol{\Omega}^{\prime}\right)=\frac{N\left(\mathbf{x}, \mathbf{x}^{\prime}\right)}{4 \pi}+\frac{3}{(4 \pi)^{2}} \boldsymbol{\Omega} \cdot \boldsymbol{\phi}\left(\mathbf{x}, \mathbf{x}^{\prime}\right) & \\
& +\frac{3}{(4 \pi)^{2}} \boldsymbol{\Omega}^{\prime} \cdot \boldsymbol{\phi}^{\prime}\left(\mathbf{x}^{\prime}, \mathbf{x}\right)+\frac{9}{(4 \pi)^{2}} \boldsymbol{\Omega} \boldsymbol{\Omega}^{\prime}: \underline{\mathbf{Q}}\left(\mathbf{x}, \mathbf{x}^{\prime}\right) .
\end{aligned}
$$

Applying these expansions to equations (3)-(7), and assuming that all neutron densities have reached their steady-state limits, we obtain the following system of equations [equations (15), (16), (17), (19), (21), (22), (23), (25)-(27)]

$$
\frac{\partial}{\partial t} N^{D}(\mathbf{x}, t)=r_{D}(\mathbf{x}) H(t) N^{N}(\mathbf{x}),
$$

where $\quad H(t)=0$ for $t<0,=1$ for $t \geq 0$, and $\frac{\mathrm{d}}{\mathrm{d} t} H(t)=\delta(t)$.

$$
\begin{aligned}
\frac{\partial}{\partial t} N^{D D}\left(\mathbf{x}, \mathbf{x}^{\prime}, t\right)= & r_{D}(\mathbf{x}) H(t) N^{N D}\left(\mathbf{x}, \mathbf{x}^{\prime}, t\right)+r_{D}\left(\mathbf{x}^{\prime}\right) H(t) N^{N D}\left(\mathbf{x}^{\prime}, \mathbf{x}, t\right) \\
& +r_{D}(\mathbf{x}) \delta\left(\mathbf{x}-\mathbf{x}^{\prime}\right) H(t) N^{*}(\mathbf{x}) . \\
{\left[\frac{\partial}{\partial t}+a\right] N^{N D}\left(\mathbf{x}, \mathbf{x}^{\prime}, t\right)=} & r_{D}\left(\mathbf{x}^{\prime}\right) N^{N y}\left(\mathbf{x}, \mathbf{x}^{\prime}, t\right) H(t)+S(\mathbf{x}) N^{D}\left(\mathbf{x}^{\prime}, t\right) \\
& -r_{D}\left(\mathbf{x}^{\prime}\right) H(t) \delta\left(\mathbf{x}-\mathbf{x}^{\prime}\right) N^{N v}(\mathbf{x})-\langle j\rangle \nabla \cdot \boldsymbol{\phi}^{N D}\left(\mathbf{x}, \mathbf{x}^{\prime}, t\right),
\end{aligned}
$$




$$
\begin{aligned}
& \text { where } \\
& \qquad \begin{array}{c}
{\left[\frac{\partial}{\partial t}+b\right] \boldsymbol{\phi}^{N D}\left(\mathbf{x}, \mathbf{x}^{\prime}, t\right)=r_{D}-\langle j\rangle r_{f^{\prime}}} \\
\\
\text { where }
\end{array} \\
& -r_{D}\left(\mathbf{x}^{\prime}\right) H(t) H(t) \delta\left(\mathbf{x}-\mathbf{x}^{\prime N}\right) \boldsymbol{\phi}^{N}\left(\mathbf{x}, \mathbf{x}^{\prime}\right) \\
& b \equiv r_{a}+(1-\langle\mu\rangle) r_{s},
\end{aligned}
$$

and $\langle\mu\rangle$ is the first moment of the scattering frequency.

$$
\begin{aligned}
& a N^{N}(\mathbf{x})=S(\mathbf{x})-\langle v\rangle \nabla \cdot \boldsymbol{\phi}^{N}(\mathbf{x}) \\
& b \boldsymbol{\phi}^{N}(\mathbf{x})=\frac{\langle v\rangle}{3} \nabla N^{N}(\mathbf{x}) . \\
& 2 a N^{N N}\left(\mathbf{x}, \mathbf{x}^{\prime}\right)=S(\mathbf{x}) N^{N}\left(\mathbf{x}^{\prime}\right)+S\left(\mathbf{x}^{\prime}\right) N^{N}(\mathbf{x})+\delta\left(\mathbf{x}-\mathbf{x}^{\prime}\right)\left[C N^{N}(\mathbf{x})+S(\mathbf{x})\right] \\
& -\langle v\rangle \boldsymbol{\nabla} \cdot \boldsymbol{\phi}^{N N}\left(\mathbf{x}, \mathbf{x}^{\prime}\right)-\langle v\rangle \boldsymbol{\nabla}^{\prime} \cdot \boldsymbol{\phi}^{N N}\left(\mathbf{x}, \mathbf{x}^{\prime}\right), \\
& C=a+\langle j(j-1)\rangle r_{f} . \\
& (a+b) \boldsymbol{\phi}^{N N}\left(\mathbf{x}, \mathbf{x}^{\prime}\right)=S\left(\mathbf{x}^{\prime}\right) \boldsymbol{\phi}^{N}(\mathbf{x})+\delta\left(\mathbf{x}-\mathbf{x}^{\prime}\right) a \boldsymbol{\phi}^{N}(\mathbf{x}) \\
& -\frac{\langle\boldsymbol{v}\rangle}{3} \boldsymbol{\nabla} N^{N N}\left(\mathbf{x}, \mathbf{x}^{\prime}\right)-\langle v\rangle \boldsymbol{\nabla}^{\prime} \cdot \underline{\mathbf{Q}}\left(\mathbf{x}, \mathbf{x}^{\prime}\right) \\
& (a+b) \boldsymbol{\phi}^{\prime N N}\left(\mathbf{x}, \mathbf{x}^{\prime}\right)=S(\mathbf{x}) \boldsymbol{\phi}^{N}\left(\mathbf{x}^{\prime}\right)+\delta\left(\mathbf{x}-\mathbf{x}^{\prime}\right) a \boldsymbol{\phi}^{N}(\mathbf{x}) \\
& -\frac{\langle\boldsymbol{v}\rangle}{3} \boldsymbol{\nabla}^{\prime} N^{N N}\left(\mathbf{x}, \mathbf{x}^{\prime}\right)-\langle v\rangle \nabla \cdot \underline{\mathbf{Q}}\left(\mathbf{x}, \mathbf{x}^{\prime}\right) . \\
& 2 b \underline{\mathbf{Q}}\left(\mathbf{x}, \mathbf{x}^{\prime}\right)=\frac{1}{3} \underline{I} \delta\left(\mathbf{x}-\mathbf{x}^{\prime}\right)\left[C^{\prime} N^{N}(\mathbf{x})+S(\mathbf{x})\right] \\
& -\frac{\langle v\rangle}{3} \boldsymbol{\nabla} \boldsymbol{\phi}^{N N}\left(\mathbf{x}, \mathbf{x}^{\prime}\right)-\frac{\langle v\rangle}{3} \nabla^{\prime} \boldsymbol{\phi}^{N N}\left(\mathbf{x}, \mathbf{x}^{\prime}\right),
\end{aligned}
$$

where

$$
C^{\prime} \equiv 2 b-r_{a}+\left\langle j^{2}\right\rangle r_{f}
$$

and $\mathbf{I}$ is the identity dyadic.

\section{INFINITE REACTOR MODEL}

It is instructive to consider first an infinite homogenous reactor. The reactor is assumed to be in the steady state in the sense that the external neutron sources are assumed time independent, and the neutron singlet and doublet densities will have reached their asymptotic values. Moreover, we assume that the detector does not perturb the neutron distribution.

This infinite reactor is described by equations (15)-(17). In view of the translational and rotational invariance we have:

(i) The neutron singlet density is independent of position, and thus the singlet current $\boldsymbol{\Phi}^{N}(\mathbf{x})$ is zero.

(ii) The neutron doublet density depends upon spatial variables only through the magnitude of the vector $\mathbf{x}-\mathbf{x}^{\prime}$. 
The solution of these equations for $N^{N}, N^{N N}\left(\mathbf{x}, \mathbf{x}^{\prime}\right)$, and $\langle v\rangle \nabla \cdot \boldsymbol{\phi}^{N N}\left(\mathbf{x}, \mathbf{x}^{\prime}\right)$ follows straightforwardly:

$$
\begin{gathered}
N^{N}=S / a, \\
N^{N N}\left(\mathbf{x}, \mathbf{x}^{\prime}\right)=\left\{N^{N}\right\}^{2}+\gamma N^{N} \delta\left(\mathbf{x}-\mathbf{x}^{\prime}\right)+\beta N^{N} \frac{\exp \left(-\sqrt{a / D}\left|\mathbf{x}-\mathbf{x}^{\prime}\right|\right)}{4 \pi D\left|\mathbf{x}-\mathbf{x}^{\prime}\right|}, \\
\langle v\rangle \boldsymbol{\nabla} \cdot \boldsymbol{\phi}^{N N}\left(\mathbf{x}, \mathbf{x}^{\prime}\right)=\beta N^{N} \delta\left(\mathbf{x}-\cdots \mathbf{x}^{\prime}\right) \cdots a \beta N^{N} \frac{\exp \left(-\sqrt{a / D}\left|\mathbf{x}-\mathbf{x}^{\prime}\right|\right)}{4 \pi D\left|\mathbf{x}-\mathbf{x}^{\prime}\right|},
\end{gathered}
$$

where

$$
\gamma \equiv \frac{c+c^{\prime \prime}+2 a}{2(a+b)}, \quad \beta=\frac{C+a}{2}-a \gamma, \quad \text { and } \quad D \equiv \frac{\langle v\rangle^{2}}{2 b} .
$$

Wc consider first the power spectral density (P.S.D.). Keeping the geometry of our detector quite arbitrary, we can write a generalized definition of P.S.D. for the infinite homogenous reactor as follows:

$$
\begin{aligned}
\left(\frac{2}{\Gamma^{2}}\right) \Phi\left(\mathbf{x}, \mathbf{x}^{\prime},(\omega)\right)= & \int_{0}^{\infty} \mathrm{d} t \cos \omega t \frac{\partial^{2}}{\partial t^{2}} \mathrm{X}^{D D}\left(\mathbf{x}, \mathbf{x}^{\prime}, t\right) \\
= & r_{D}(\mathbf{x})\left\{\mathrm{X}^{N D}\left(\mathbf{x}, \mathbf{x}^{\prime}, i \omega\right)-\overline{\mathrm{X}}^{N D}\left(\mathbf{x}, \mathbf{x}^{\prime},-i \omega\right)\right\} i \omega \\
& +r_{D}(\mathbf{x}) \delta\left(\mathbf{x}-\mathbf{x}^{\prime}\right) N^{N},
\end{aligned}
$$

where $\overrightarrow{\mathrm{X}}^{N D}\left(\mathbf{x}, \mathbf{x}^{\prime}, s\right)$ is the Laplace transform of the neutron-detected particle correlation function (i.e. $\left.N^{V D}\left(\mathbf{x}, \mathbf{x}^{\prime}, t\right)-N^{N}(\mathbf{x}) N^{D}\left(\mathbf{x}^{\prime}, t\right)\right)$. We have made use of equation (11) and the fact that in an infinite reactor

$$
r_{D}(\mathbf{x}) N^{N D}\left(\mathbf{x}, \mathbf{x}^{\prime}, t\right)=r_{D}\left(\mathbf{x}^{\prime}\right) N^{N D}\left(\mathbf{x}^{\prime}, \mathbf{x}, t\right) .
$$

In addition we have utilized the relationship between the cosine and Laplace transforms:*

$$
F^{\text {C.T. }}(\omega)=\frac{1}{2}\left[F^{\text {L.T. }} \cdot(i \omega)+F^{\text {L.T. }}(-i \omega)\right]
$$

Applying the definitions of $\mathrm{X}^{N D}\left(\mathbf{x}, \mathbf{x}^{\prime}, t\right)$ and the neutron correlation function $\left(N^{N N}\left(\mathbf{x}, \mathbf{x}^{\prime}\right)-N^{N} N^{N}\right)$ to (17) and (19) we find that:

$$
\begin{aligned}
{\left[\frac{\partial}{\partial t}+a\right] \mathrm{X}^{N D}\left(\mathbf{x}, \mathbf{x}^{\prime}, t\right)=} & r_{D}\left(\mathbf{x}^{\prime}\right) H(t) \mathrm{X}^{N \nu}\left(\mathbf{x}, \mathbf{x}^{\prime}\right) \\
& -r_{D}\left(\mathbf{x}^{\prime}\right) H(t) \delta\left(\mathbf{x}-\mathbf{x}^{\prime}\right) N^{N}-\langle v\rangle \nabla \cdot \boldsymbol{\phi}^{N D}\left(\mathbf{x}, \mathbf{x}^{\prime}, t\right)
\end{aligned}
$$

and

$$
\left[\frac{\partial}{\partial t}+b\right] \boldsymbol{\phi}^{N D}\left(\mathbf{x}, \mathbf{x}^{\prime}, t\right)=r_{D}\left(\mathbf{x}^{\prime}\right) H(t) \boldsymbol{\phi}^{N N}\left(\mathbf{x}, \mathbf{x}^{\prime}\right)-\frac{\langle v\rangle}{3} \nabla \mathrm{X}^{N D}\left(\mathbf{x}, \mathbf{x}^{\prime}, t\right) .
$$

The solution of the transform of equations (36) and (37) for $\overline{\mathrm{X}}^{N D}(\mathbf{x}, \mathbf{x}, s)$ follows in a straightforward manner with the application of (30), (31) and the boundary conditions $\mathrm{X}^{N D}\left(\mathbf{x}, \mathbf{x}^{\prime}, 0\right)=0$ and $\phi^{N D}\left(\mathbf{x}, \mathbf{x}^{\prime}, 0\right)=0$.

We find that

$$
\begin{aligned}
& \overline{\mathrm{X}}^{N D}\left(\mathbf{x}, \mathbf{x}^{\prime}, s\right)=\frac{r_{D}\left(\mathbf{x}^{\prime}\right) \beta N^{N} \exp \left(-\sqrt{a / D}\left|\mathbf{x}-\mathbf{x}^{\prime}\right|\right)}{s^{2} 4 \pi D\left|\mathbf{x}-\mathbf{x}^{\prime}\right|} \\
& -\frac{r_{D}\left(\mathbf{x}^{\prime}\right)(s+b)[\beta-s(\gamma-1)] N^{N} \exp \left(-\sqrt{(s+a)(s+b) / b D}\left|\mathbf{x}-\mathbf{x}^{\prime}\right|\right)}{s^{2} 4 \pi D b\left|\mathbf{x}-\mathbf{x}^{\prime}\right|} .
\end{aligned}
$$

* This is obviously valid whenever the integral for the cosine transform converges, and is, in any event, correct in the sense of generalized functions. 
Equations (33) and (38) provide us with a solution for the generalized P.S.D. in an infinite reactor. Analysis of both single and double detector experiments is possible. In accordance with our primary aim of understanding the specific experiments mentioned in Section 1, we shall restrict ourselves to a single detector.

The P.S.D. is obtained by integrating the r.h.s. of (33) over the detector volume [see equation (3)]. For a spherical detector of radius $R$, we obtain:

$$
\left(\frac{2}{\Gamma^{2}}\right) \Phi(\omega)=r_{D}\left\{\overline{\mathrm{X}}^{N D}(R, i \omega)-\overline{\mathrm{X}}^{N D}(R,-i \omega)\right\} i \omega+r_{D} N^{N} V_{D}
$$

where $V_{\nu}-\left(4 \pi R^{3} / 3\right)$ is the detector volume, and

$$
\begin{aligned}
\overline{\mathrm{X}}^{N D}(R, s)= & \frac{r_{D} \beta N^{N} R^{5}}{s^{2} 4 \pi D} G\left(\sqrt{\frac{a}{D}} R\right) \\
& -\frac{r_{D}(s+b)[\beta-s(\gamma-1)] N^{N} R^{5}}{s^{2} 4 \pi D b} G\left(\sqrt{\frac{(s+a)(s+b)}{b D}} R\right) .
\end{aligned}
$$

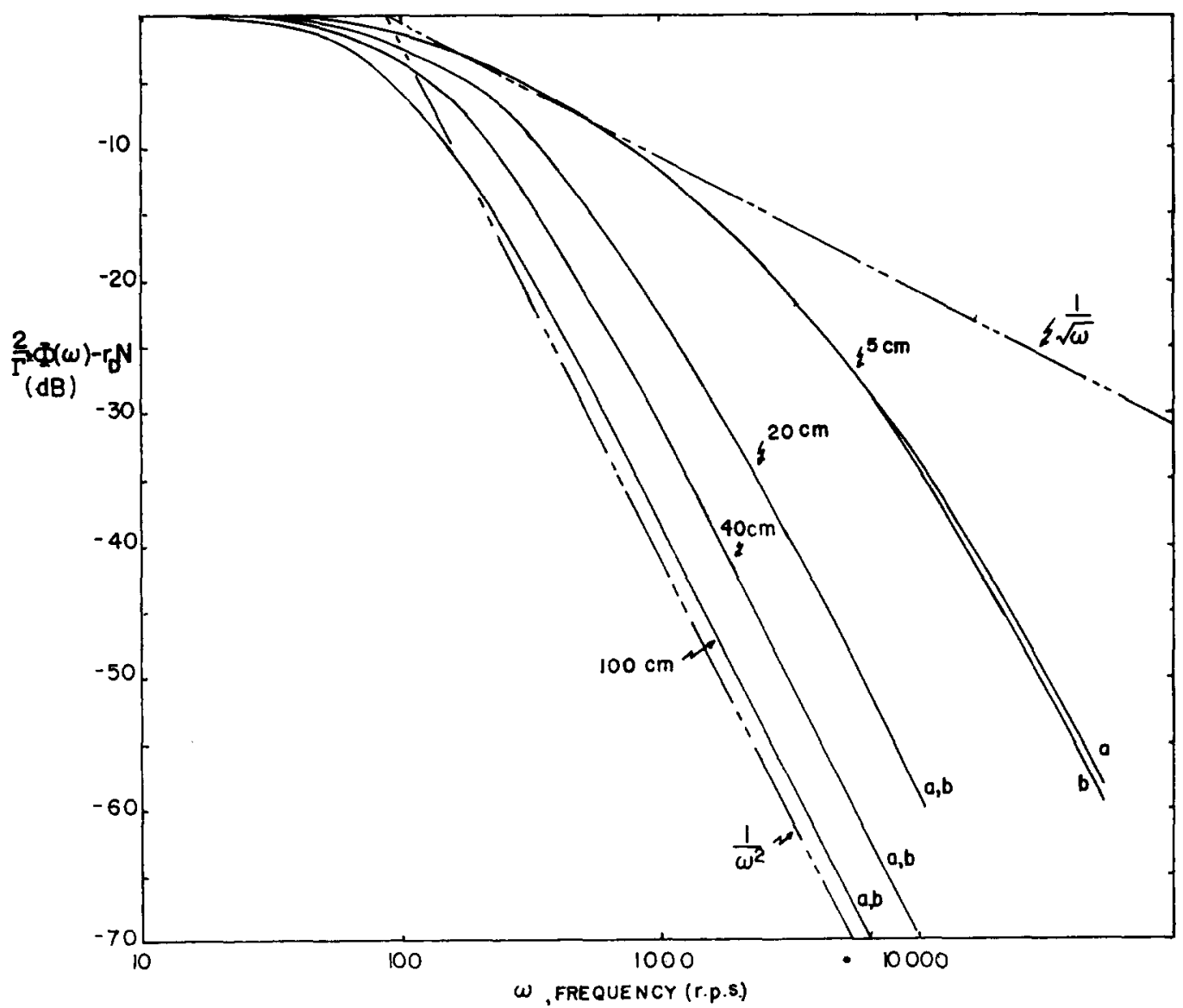

FIG. 1.-Characteristic frequency dependence of the P.S.D. as calculated for spherical detectors of various radii in an infinite water moderated-reactor $\left(r_{a}=8000 \mathrm{sec}^{-1}\right.$, $k_{\infty}=.99$, and $D=36,590 \mathrm{~cm}^{2} / \mathrm{sec}$ ). Calculations $a$ are carried out with equations (38) and (48), calculations $b$ with equations (38) and (39). The asymptotic infinite detector frequency dependence $\left(1 / \omega^{2}\right)$ is displayed along with the asymptotic point detector dependence $\left(1 / \omega^{1 / 2}\right)$. 


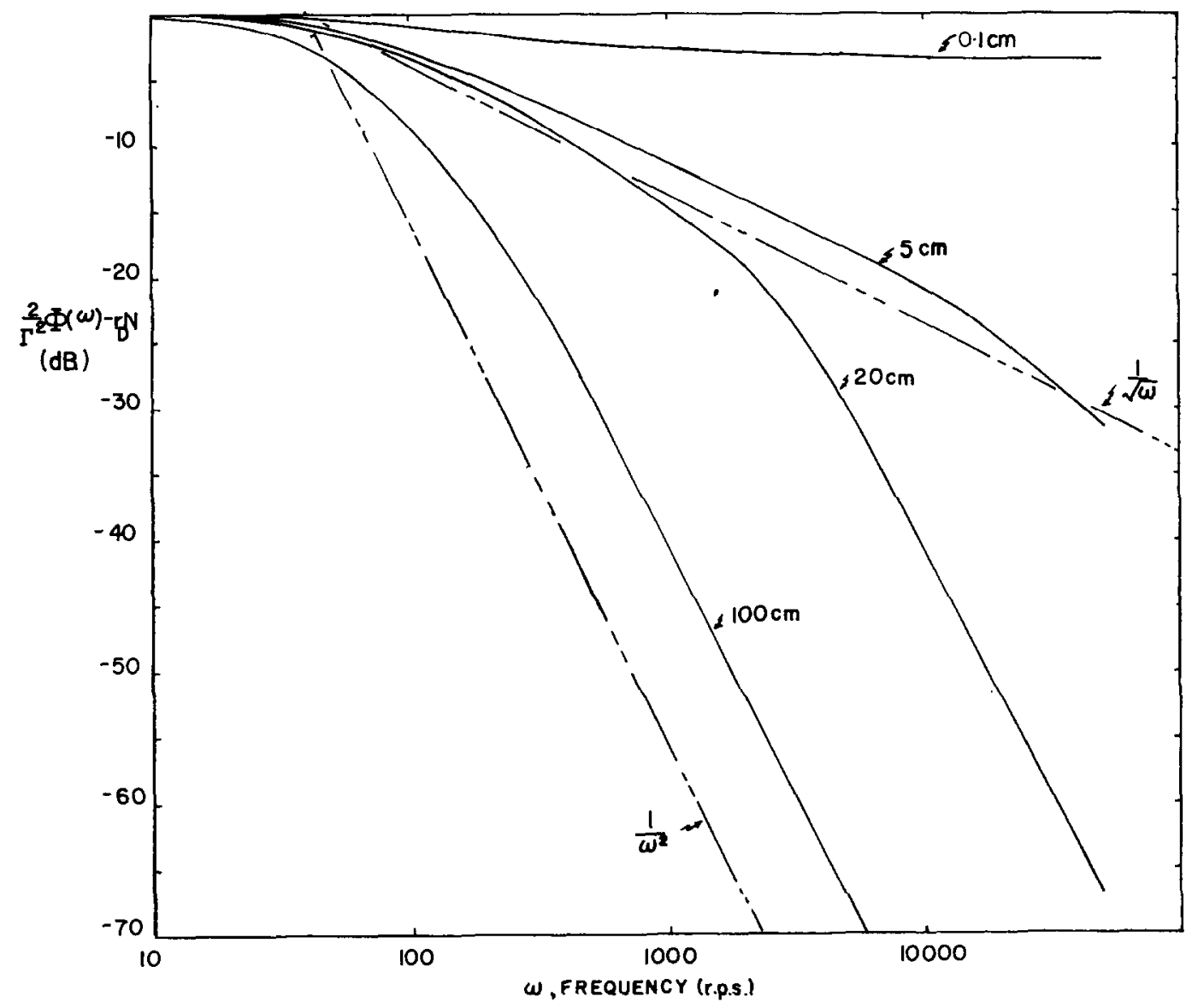

FIG. 2.-Characteristic frequency dependence of the P.S.D. as calculated for spherical detectors of various radii in an infinite graphite-moderated reactor $\left(r_{a}-1000 \mathrm{sec}^{-1}\right.$, $k_{\infty}=-96$, and $D=182,954 \mathrm{~cm}^{2} / \mathrm{sec}$ ). The calculations are performed using equations (38) and (39).

The integral $G(\lambda R)$ is given by

$$
G(\lambda R)=\frac{1}{R^{5}} \int_{|\mathbf{x}| \leq R} \mathrm{~d}^{3} \mathbf{x} \int_{\left|\mathbf{x}^{\prime}\right| \leq R} \mathrm{~d}^{3} \mathbf{x}^{\prime} \frac{\mathrm{e}^{-\lambda\left|\mathbf{x}_{-} \mathbf{x}^{\prime}\right|}}{\left|\mathbf{x}-\mathbf{x}^{\prime}\right|}=8 \pi^{2}\left[\frac{2}{3} \frac{1}{(\lambda R)^{2}}-\frac{1}{(\lambda R)^{5}} F(\lambda R)\right],
$$

where

$$
F(\lambda R)=(1+\lambda R)^{2} \mathrm{e}^{-2 \lambda R}+(\lambda R)^{2}-1 .
$$

The quantity $\left(2 / \Gamma^{2}\right) \Phi(\omega)-r_{D} N^{N} V_{D}$ is plotted in Figs. 1 and 2 for water and graphitemoderated systems for a number of detector radii. However, before discussing these results, we should make note of two limiting cases for $\Phi(\omega)$.

The first, which provides a check with the space and energy-independent theory is the large detector limit. For $R$ such that

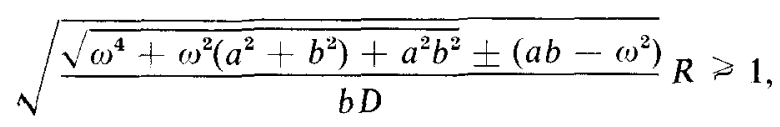


we find that

and the P.S.D. is given by:

$$
G(\lambda R) \cong \frac{(4 \pi)^{2}}{3(\lambda R)^{2}}
$$

$$
\left(\frac{2}{\Gamma^{2}}\right) \Phi(\omega) \cong r_{D} N^{N} V_{D}+r_{D}{ }^{2} N^{N} V_{D} \frac{\langle j(j-1)\rangle r_{f}}{\left(a^{2}+\omega^{2}\right)}
$$

This is the well-known space and energy-independent theory result. However, it must be pointed out that conditions ( $43 \pm$ ) are met for a detector of large but finite radius, given $a$ and $b$, only for frequencies greater than some $\omega_{0}$ [which can be determined from the condition (43-)].

The second limit is for the small or 'point' detector. Here if

$$
\sqrt{\frac{\sqrt{\omega^{4}+\omega^{2}\left(a^{2}+b^{2}\right)+a^{2} b^{2}} \pm\left(a b-\omega^{2}\right)}{b D}} R \leqslant 1 .
$$

Then

and the P.S.D. becomes

$$
G(\lambda R) \cong\left(\frac{4 \pi}{3}\right)^{2}\left[\frac{9}{15}-\lambda R\right]
$$

$$
\begin{aligned}
\left(\frac{2}{\Gamma^{2}}\right) \Phi(\omega) \cong & r_{D} N^{N} V_{D}+r_{D}{ }^{2} N^{N} V_{D}{ }^{2} \frac{[\gamma-1-\beta / b]}{2 \pi D R} \\
& +\frac{r_{D}{ }^{2} N^{N} V_{D}^{2} \beta}{2 \pi D} \sqrt{\frac{\sqrt{\omega^{4}+\omega^{2}\left(a^{2}+b^{2}\right)+a^{2} b^{2}}-a b+\omega^{2}}{b D}}
\end{aligned}
$$

As before, conditions ( $46 \pm$ ) are met for some range of frequencies for a given $R, a$ and $b$. Here this range includes all frequencies less than some $\omega_{0}$ determined by condition $(46+)$. The P.S.D., $\Phi(\omega)$, is independent of frequency for $R$ sufficiently small that the first and second terms on the r.h.s. of (47) dominate. For $R$ such that the third term is dominant, we find that when $a<\omega<b$, which is the frequency range of experimental interest, $\Phi(\omega)$ goes as $\omega^{-1 / 2}$ (for all thermal reactors $b \gg a$ ).

In Figs. 1 and 2 both limiting cases [(45) and (48)] of $\Phi(\omega)$ are exhibited. In addition it can be seen that for intermediate values of $R$ the P.S.D. displays the expected combination of behaviours, point-detector-like for low frequencies and as a large detector for higher frequencies.

In Fig. 1 we have also plotted $\Phi(\omega)$ as computed by a much simpler approximation scheme. Noting that $b$ (the transport or scattering rate) is much greater than all other rates (namely $r_{a}, r_{f}$ and $\omega$ in the range of experimental interest) we can take the ratios of these rates with $b$ to be zero. Expression (40) for the power spectral density is thus greatly simplified as

$$
\mathrm{X}^{N D}(R, s) \cong \frac{r_{D}\langle j(j-1)\rangle r_{f} N^{N} R^{5}}{8 \pi D s^{2}}\left[G\left(\sqrt{\frac{a}{D}} R\right)-G\left(\sqrt{\frac{s+a}{D}} R\right)\right] .
$$

This approximation can also be applied to the working equations by ignoring terms containing ratios of the various other rates to $b$ (the transport rate): i.e.

$$
\frac{1}{b} \frac{\partial}{\partial t} \approx 0, \quad \frac{1}{b} D \nabla^{2} \approx 0, \quad \frac{r_{a}}{b} \approx 0, \text { and } \frac{r_{f}}{b} \approx 0
$$


Applying these conditions, we find that equations (19), (20), (25), (26) and (27) become respectively:

$$
\begin{gathered}
\boldsymbol{\phi}^{N D}\left(\mathbf{x}, \mathbf{x}^{\prime}, t\right)=-\frac{D}{\langle v\rangle} \nabla N^{N D}\left(\mathbf{x}, \mathbf{x}^{\prime}, t\right), \\
\boldsymbol{\phi}^{N}(\mathbf{x})=-\frac{D}{\langle v\rangle} \nabla N^{N}(\mathbf{x}), \\
\boldsymbol{\phi}^{N N}\left(\mathbf{x}, \mathbf{x}^{\prime}\right)=-\frac{D}{\langle v\rangle} \boldsymbol{\nabla} N^{N N}\left(\mathbf{x}, \mathbf{x}^{\prime}\right) \cdots \frac{3 D}{v v} \nabla^{\prime} \cdot \underline{\mathbf{Q}}\left(\mathbf{x}, \mathbf{x}^{\prime}\right), \\
\boldsymbol{\phi}^{N N}\left(\mathbf{x}, \mathbf{x}^{\prime}\right)=-\frac{D}{\langle v\rangle} \nabla^{\prime} N^{N N}\left(\mathbf{x}, \mathbf{x}^{\prime}\right)-\frac{3 D}{v v\rangle} \boldsymbol{\nabla} \cdot \underline{\mathbf{Q}}\left(\mathbf{x}, \mathbf{x}^{\prime}\right),
\end{gathered}
$$

and

$$
\underline{\mathbf{Q}}=\frac{1}{3} \underline{\mathbf{I}} \delta\left(\mathbf{x}-\mathbf{x}^{\prime}\right) N^{N}(\mathbf{x}) .
$$

From Fig. 1 it is clear that the new approximation scheme is very good as compared to our more general scheme. Since we will always have in mind systems in which the scattering rate is much larger than all other pertinent rates, we shall use (51)-(55) in the remaining analysis. It should be pointed out that this scheme corresponds to that used by AKCasu (to be published); Mathes (1962); Harris, and SheFF in their inductive approaches to these problems. SHefF has reported results for a cubical detcetor of arbitrary size in an infinite reactor in terms of the auto-correlation function and for the P.S.D. in the point detector limit. Agreement between their results and those presented here seems to be very good.

Finally, the most important conclusion to be drawn from Figs. 1 and 2 is that a finite spherical detector in an infinite homogenous reactor in no sense describes the particular P.S.D. density experiment which we are attempting to understand. The active volume of the detector used by Ricker et al. clearly places it in the point detector limit, and yet his experimental results (see Fig. 3) are in complete agreement with the large detęctor limit.

Before giving up the infinite reactor description, we must look at the possibility of detector geometry being an important effect. Of primary interest will be a long thin cylinder, as this is the geometry of the detector employed in the experiment of Ricker et al. Computing the P.S.D. for such a detector is straightforward in the new approximation scheme. We will use the generalized definition [equation (33)] of the P.S.D. but first we insert (51) into (17) to obtain

$$
\left[\frac{\partial}{\partial t}+a-D \nabla^{2}\right] \mathrm{X}^{N D}\left(\mathbf{x}, \mathbf{x}^{\prime}, t\right)=r_{D}\left(\mathbf{x}^{\prime}\right) H(t) \mathrm{X}^{N \alpha}\left(\mathbf{x}, \mathbf{x}^{\prime}\right)-r_{D}\left(\mathbf{x}^{\prime}\right) H(t) \delta\left(\mathbf{x}-\mathbf{x}^{\prime}\right) N^{N}
$$

where

and

$$
\mathrm{X}^{N N}\left(\mathbf{x}, \mathbf{x}^{\prime}\right)=N^{N N}\left(\mathbf{x}, \mathbf{x}^{\prime}\right)-\left\{N^{N}\right\}^{2}
$$

$N^{N N}\left(\mathbf{x}, \mathbf{x}^{\prime}\right)=\left\{N^{N}\right\}^{2}+\delta\left(\mathbf{x}-\mathbf{x}^{\prime}\right) N^{N}+\frac{\langle j(j+1)\rangle r_{f} N^{N} \exp \left(-\sqrt{a / D}\left|\mathbf{x}-\mathbf{x}^{\prime}\right|\right)}{8 \pi D\left|\mathbf{x}-\mathbf{x}^{\prime}\right|}$. 
[Note that (30) reduces to (58) simply by letting $b$ go to infinity.] Solving equation (56) for $\mathrm{X}^{N D}\left(\mathbf{x}, \mathbf{x}^{\prime}, t\right)$ we find that:

$$
\begin{aligned}
& \left(\frac{2}{\Gamma^{2}}\right) \Phi\left(\mathbf{x}, \mathbf{x}^{\prime}, \omega\right)=r_{D}\left(\mathbf{x}^{\prime}\right) \delta\left(\mathbf{x}-\mathbf{x}^{\prime}\right) N^{\nu} \\
& +r_{D}(\mathbf{x}) r_{D}\left(\mathbf{x}^{\prime}\right) \frac{\langle j(j+1)\rangle r_{f} N^{N}}{(2 \pi)^{3}} \int \frac{\mathrm{d}^{3} \mathbf{k} \mathrm{e}^{-i \mathbf{k} \cdot\left(\mathbf{x}-\mathbf{x}^{\prime}\right)}}{\left(a+D k^{2}\right)^{2}+\omega^{2}},
\end{aligned}
$$

where we have used the identity:

$$
\frac{\exp \left(-\sqrt{a / D}\left|\mathbf{x}-\mathbf{x}^{\prime}\right|\right)}{4 \pi D\left|\mathbf{x}-\mathbf{x}^{\prime}\right|}=\frac{1}{(2 \pi)^{3}} \int \frac{\mathrm{d}^{3} \mathbf{k} \mathrm{e}^{-i \mathbf{k} \cdot\left(\mathbf{x}-\mathbf{x}^{\prime}\right)}}{\left(a+D k^{2}\right)^{2}+\omega^{2}} .
$$

The integration of $\Phi\left(\mathbf{x}, \mathbf{x}^{\prime}, \omega\right)$ over any single detector is facilitated by noting that:

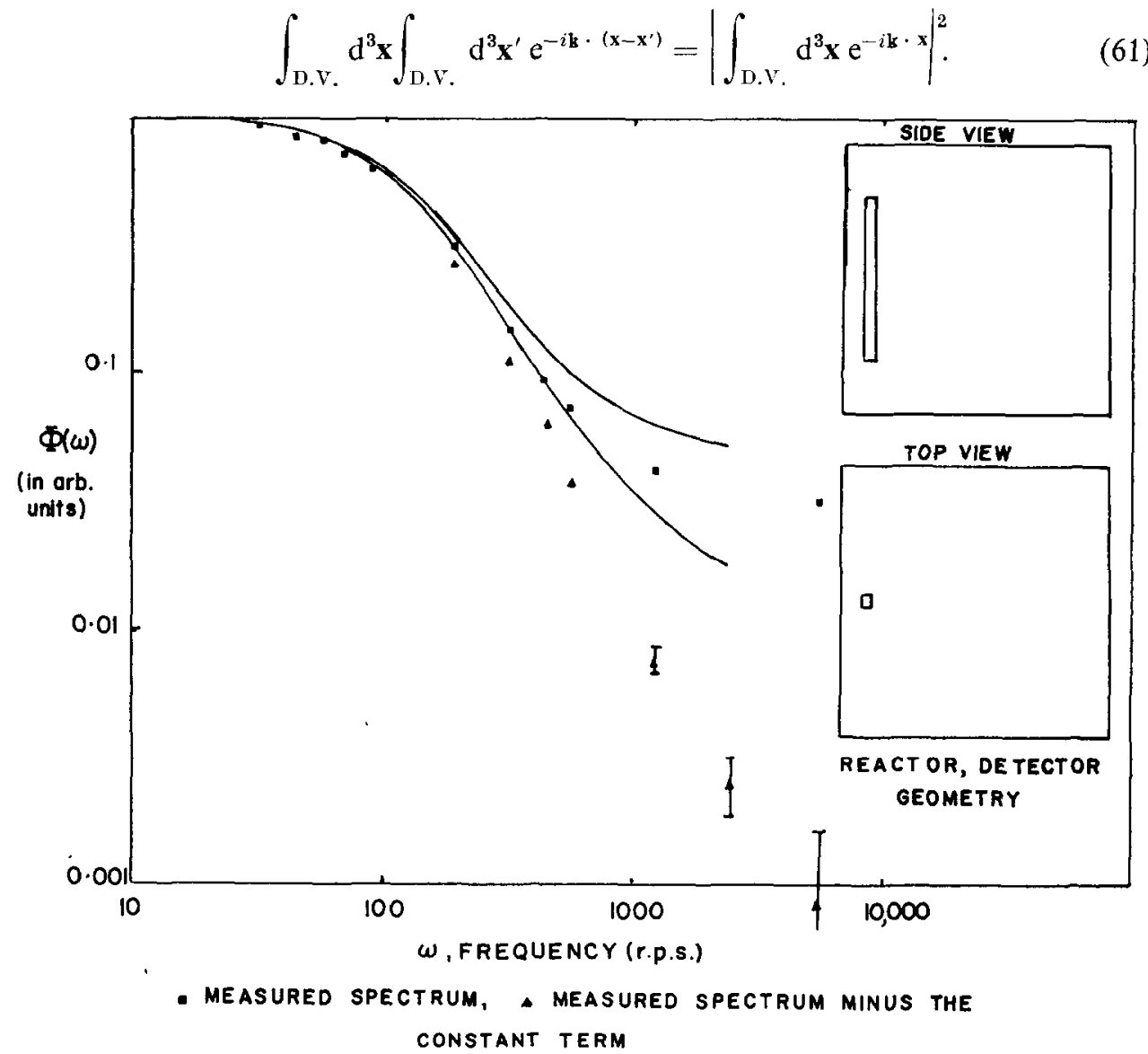

FIG. 3.- Measured and calculated P.S.D. for a small water-moderated reactor near critical $\beta / \Lambda=117 \mathrm{sec}^{-1}, L=2.88 \mathrm{~cm}$. The solid squares are the measured values (by RICKER et al.) for the P.S.D. The upper curve is the P.S.D. as calculated by equation (85) with $D=r_{a} L^{2}$. The solid triangles represent the P.S.D. minus the constant term as obtained from the measured data by subtracting an appropriate constant. The lower curve is the P.S.D. minus the constant term as calculated from equation (85). The reactor detector geometry is chosen to represent the experiment (a $50 \mathrm{~cm}$ on a side homogenous bare reactor, and a $2 \times 2 \mathrm{~cm} \times 30 \mathrm{~cm}$ rectangular parallel-piped detector whose outer is $5 \mathrm{~cm}$ in from the side of the reactor). 
The final result is simply

$$
\left(\frac{2}{\Gamma^{2}}\right) \Phi(\omega)=r_{D} N^{N} V_{D}+r_{D}^{2}\langle j(j-1)\rangle r_{f} \frac{N^{N} V_{D} R^{2}}{2 D} \frac{\tan ^{-1}(\omega / a)}{\omega}
$$

where $V_{0}=\pi R^{2} l, R$ being the detector radius, and $l$ the detector length. This approximate result is applicable for $l$ large enough that:

and $R$ small enough that:

$$
\sqrt{\frac{\sqrt{a^{2}+\omega^{2}} \pm a}{2 D}} l \gg 1
$$

$$
\sqrt{\frac{\sqrt{a^{2}+\omega^{2}} \pm a}{2 D}} R \leqslant 1
$$

Ricker's detector (a $\mathrm{BF}_{3}$ tube), which has $R \sim 3 \mathrm{~cm}$ and $l \sim 30 \mathrm{~cm}$, will satisfy the second condition (64) over the frequency range of interest, but conditions (63士) are met (using the reactor parameters in Fig. 1) only for frequencies in excess of 100 r.p.s. Thus according to this model the detector should behave like a point for frequencies somewhat less than 100 r.p.s., and as a line for frequencies greater than 100 r.p.s., but in no case like an infinite detector. Clearly the finite cylindrical detector, infinite rcactor model dosen't explain Ricker's experiment.

We have also calculated the P.S.D. for a large, thin rectangular plate detector, just as for the long, thin cylinder, and it displays a still different frequency dependence; i.e.

$$
\left(\frac{2}{\Gamma^{2}}\right) \Phi(\omega)=r_{D} N^{N} V_{l}+r_{D}{ }^{2} \frac{\langle j(j+1)\rangle r_{f} N^{N} V_{I}}{2 \sqrt{2 D}} l_{1}\left\{\frac{1}{\sqrt{a^{2}+\omega^{2}} \sqrt{\sqrt{a^{2}+\omega^{2}}+a}}\right\},
$$

where

$$
\sqrt{\frac{V_{D}=l_{1} l_{2} l_{3},}{2 D}} l_{2,3} \gg 1
$$

and

$$
\sqrt{\frac{\sqrt{a^{2}+()^{2}} \pm a}{2 D}} l_{1} \ll 1 .
$$

In Table 1 the characteristic frequency dependence $\left(2 / \Gamma^{2}\right) \Phi(\omega)-r_{0} N^{N} V_{0}$ near critical $a \approx 0$ of the P.S.D.'s, for each of the limiting cases of detector geometry that have been considered, is displayed. These results argue strongly that knowledge of

TABle 1.-Characteristic FrequenCy DePendenCe OF THE P.S.D. NEAR CRITICAL $(a \approx 0)$ FOR VARIOUS DETECTOR GEOMETRIES

\begin{tabular}{ccccc}
\hline $\begin{array}{c}\text { Detector } \\
\text { geometry }\end{array}$ & $\begin{array}{c}\text { Small } \\
\text { sphere }\end{array}$ & $\begin{array}{c}\text { Tong thin } \\
\text { cylinder }\end{array}$ & $\begin{array}{c}\text { Thin } \\
\text { plate }\end{array}$ & $\begin{array}{c}\text { I.arge } \\
\text { sphere }\end{array}$ \\
\hline$\frac{2}{\Gamma^{2}} \Phi(\omega)-r_{D} N$ & $\frac{1}{\sqrt{\omega}}$ & $\frac{1}{\omega}$ & $\frac{1}{\omega^{3 / 2}}$ & $\frac{1}{\omega^{2}}$ \\
\hline$\frac{\Gamma^{2}}{} \Phi(0)-r_{D} N$ & & & & \\
\hline
\end{tabular}


the manner in which the neutron doublet density (the density of correlated neutrons) is sampled by a detector is very important to the interpretation of any experiment which purports to measure neutron noise phenomena. Therefore we are forced to conclude that in the case of Ricker's P.S.D. measurements considerations other than detector geometry must play an even more important role. The role of finite reactor size and geometry is logically the next to be investigated. However, before proceeding with this, we must look at the companion experiment, the measurement of the variance-to-mean with a finite detector in an infinite reactor.

The definition of the variance-to-mean (4) for an arbitrary detector in an infinite reactor becomes:

$$
V / M=\int_{\mathrm{D} . \mathrm{V} .} \mathrm{d}^{3} \mathbf{x} \int_{\mathrm{D}, \mathrm{V} .} \mathrm{d}^{3} \mathbf{x}^{\prime} \mathrm{X}^{D D}\left(\mathbf{x}, \mathbf{x}^{\prime}, t\right) / r_{D} N^{N} V_{D} t .
$$

In order to find $\mathrm{X}^{D D}\left(\mathbf{x}, \mathbf{x}^{\prime}, t\right)$ we make use again of the solution of equation (56) for $\mathrm{X}^{N D}\left(\mathbf{x}, \mathbf{x}^{\prime}, t\right)$. The result is

$$
\begin{aligned}
& \mathbf{X}^{D D}\left(\mathbf{x}, \mathbf{x}^{\prime}, t\right)=r_{D}\left(\mathbf{x}^{\prime}\right) \delta\left(\mathbf{x}-\mathbf{x}^{\prime}\right) N^{N} t
\end{aligned}
$$

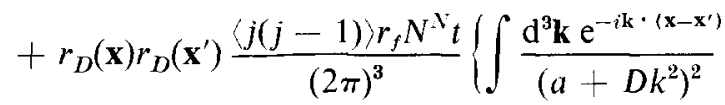

$$
\begin{aligned}
& \left.+\int \mathrm{d}^{3} k \frac{\left(1-\mathrm{e}^{-\left(a+D k^{2}\right) t}\right)}{\left(a+D k^{2}\right)^{3} t} \mathrm{e}^{-i \mathbf{k} \cdot\left(\mathbf{x}-\mathbf{x}^{\prime}\right)}\right\}
\end{aligned}
$$

If we perform integrals over $\mathbf{x}$ and $\mathbf{x}^{\prime}$ for an infinite detector (one whose radius is such that $\sqrt{a / d} R \gg 1$, we get the following result for the variance-to-mean:

$$
(V / M)_{\text {large detector }}=1+\frac{r_{D}}{r_{a}} \frac{\langle j(j-1)\rangle k_{\infty}}{\langle j\rangle\left(1-k_{\infty}\right)^{2}}\left[1-\frac{1-\mathrm{e}^{-a t}}{a t}\right],
$$

where $k_{\infty} \equiv\left(\langle j\rangle r_{f} / r_{a}\right)$ is the infinite medium multiplication factor. This is the spaceenergy-independent theory result to which Albrecht was able to fit his variance-tomean measurements. However, Albrecht's detector is small (in the sense that $\sqrt{a / d} R \ll 1)$ so that we should properly integrate $\mathrm{X}^{D I}\left(\mathbf{x}, \mathbf{x}^{\prime}, t\right)$ only over the finite detector volume. When this is done we obtain a complicated expression for $V / M$. For the sake of comparison, it is sufficient to consider the long time limit, which gives:

$$
\lim _{t \rightarrow \infty} \frac{(V / M-1)_{\text {Small Detector }}}{(V / M-1)_{\text {Large Detector }}}=\frac{1}{6}\left(\sqrt{\frac{a}{D}} R\right)^{3} .
$$

This is small $\left(\sim 1 / 10^{3}\right)$ for parameters appropriate to Albrecht's experiment. Thus we see that we are in exactly the same uncomfortable position with respect to explaining a variance-to-mean measurement in terms of a finite detector, infinite reactor system as we were with the P.S.D. measurements.

\section{FINITE REACTOR MODEL}

We attack the problem of the finite reactor in the approximation scheme of equations (51)-(55) by an expansion in the eigenfunctions of the Laplacian operator appropriate to the geometry of the system. Singling out these eigenfunctions which are 
zero on the extrapolated boundaries of the reactor, we can define the following complete orthonormal set:

$$
\begin{gathered}
\nabla^{2} \psi_{n}(\mathbf{x})=-B_{n}^{2} \psi_{n}(\mathbf{x}), \\
\int_{\text {R.V. }} \mathrm{d}^{\mathbf{3} \mathbf{x}} \psi_{n}(\mathbf{x}) \psi_{m}(\mathbf{x})=\delta_{n m}, \\
\sum_{n=1}^{\infty} \psi_{n}(\mathbf{x}) \psi_{n}\left(\mathbf{x}^{\prime}\right)=\delta\left(\mathbf{x}-\mathbf{x}^{\prime}\right),
\end{gathered}
$$

where R.V. = reactor volume. The functions in this set are sometimes referred to as the reactor modes. It is convenient to use correlation functions instead of doublet densities and to treat only neutron co-ordinates in the expansion. We therefore write:

$$
\begin{aligned}
\mathrm{X}^{N D}\left(\mathbf{x}, \mathbf{x}^{\prime}, t\right) & =\sum_{n=1}^{\infty} A_{n}^{N D}\left(\mathbf{x}^{\prime}, t\right) \psi_{n}(\mathbf{x}) \\
N^{N}(\mathbf{x}) & =\sum_{n=1}^{\infty} A_{n}^{N} \psi_{n}(\mathbf{x}) \\
S(\mathbf{x}) & =\sum_{n=1}^{\infty} S_{n} \psi_{n}(\mathbf{x}) \\
\mathrm{X}^{N N}\left(\mathbf{x}, \mathbf{x}^{\prime}\right) & =\sum_{n, m=1}^{\infty} A_{n m}{ }^{N x} \psi_{n}(\mathbf{x}) \psi_{m}\left(\mathbf{x}^{\prime}\right)
\end{aligned}
$$

The solution for $A_{n}{ }^{v}$ follows immediately from equations (21) and (52)

$$
A_{n}^{N}=S_{n} /\left(a+D B_{n}{ }^{2}\right) \text {. }
$$

We have assumed that the neutron densities have achieved a steady state, and for now the spatial distribution of the external neutron sources (i.e. the $S_{n}$ 's) will remain arbitrary. $A_{n m}{ }^{N N}$ is found by taking moments of the appropriate equation for $\mathrm{X}^{N N}\left(\mathbf{x}, \mathbf{x}^{\prime}\right)$. This equation (in the new approximation scheme) is the result of combining equations (23), (53), (54) and (55):

$$
\begin{aligned}
{\left[2 a-D \nabla^{2}-D \nabla^{\prime 2}\right] \mathrm{X}^{N N}\left(\mathbf{x}, \mathbf{x}^{\prime}\right)=} & \delta\left(\mathbf{x}-\mathbf{x}^{\prime}\right)\left[C N^{N}(\mathbf{x})+S(\mathbf{x})\right] \\
& +2 D \boldsymbol{\nabla} \cdot \boldsymbol{\nabla}^{\prime} \delta\left(\mathbf{x}-\mathbf{x}^{\prime}\right) N^{N}(\mathbf{x}) .
\end{aligned}
$$

Taking the moments of $(80)$ with respect to the reactor modes we thus obtain:

where

$$
A_{n m}{ }^{N N}=\sum_{i=1}^{\infty} C_{i n m} A_{i}^{N}\left\{\frac{\left(C+a+D B_{n}{ }^{2}+D B_{m}{ }^{2}\right)}{\left(2 a+D B_{n}{ }^{2}+D B_{m}{ }^{2}\right)}\right\},
$$

$$
C_{i n m} \equiv \int \mathrm{d}^{3} \mathbf{x} \psi_{i}(\mathbf{x}) \psi_{n}(\mathbf{x}) \psi_{m}(\mathbf{x})
$$

Finally we need the equation for $A_{n}{ }^{N D}\left(\mathbf{x}^{\prime}, t\right)$. The appropriate equation for $\mathrm{X}^{N D}\left(\mathbf{x}, \mathbf{x}^{\prime}, t\right)$ is :

$$
\begin{aligned}
{\left[\frac{\partial}{\partial t}+a \rightarrow D \nabla^{2}\right] \mathrm{X}^{N D}\left(\mathbf{x}, \mathbf{x}^{\prime}, t\right)=} & r_{D}\left(\mathbf{x}^{\prime}\right) H(t) \mathrm{X}^{N N}\left(\mathbf{x}, \mathbf{x}^{\prime}\right) \\
& -r_{D}\left(\mathbf{x}^{\prime}\right) H(t) \delta\left(\mathbf{x}-\mathbf{x}^{\prime}\right) N^{N}(\mathbf{x}),
\end{aligned}
$$


where equations (17) and (51) have been combined. Proceeding as above we find immediately that:

$$
\left[\frac{\partial}{\partial t}+a+D B_{n}^{2}\right] A_{n}{ }^{N D}\left(\mathbf{x}^{\prime}, t\right)=r_{D}\left(\mathbf{x}^{\prime}\right) H(t) \sum_{i, m=1}^{\infty} C_{i n m} A_{i}\left\{\frac{(C-a)}{\left(2 a+D B_{n}{ }^{2}+D B_{m}{ }^{2}\right)}\right\} \psi_{m}\left(\mathbf{x}^{\prime}\right)
$$

The solution of this equation for $A_{n}{ }^{N D}\left(\mathbf{x}^{\prime}, t\right)$ provides us with expressions for the P.S.D., the two detector or 'cross P.S.D.', or the variance-to-mean, all for arbitrary detector location and geometry in reactors of arbitrary configuration. In the final results [for example $\Phi(\omega)$ ], geometric effects are contained in the $\Psi_{n}^{\prime}$ 's; i.e. in the set of constants $C_{i n m}$ and in integrals of the $\Psi_{n}^{\prime}$ 's over the detector volume.

For the generalized P.S.D. we find:

$$
\begin{aligned}
& \left(\frac{2}{\Gamma^{2}}\right) \Phi\left(\mathbf{x}, \mathbf{x}^{\prime}, \omega\right)=r_{D}(\mathbf{x}) \delta\left(\mathbf{x}-\mathbf{x}^{\prime}\right) N^{N}(\mathbf{x}) \\
& \left.\quad+r_{D}(\mathbf{x}) r_{D}\left(\mathbf{x}^{\prime}\right) \sum_{i, n, m=1}^{\infty} C_{i n m} A_{i}^{N} \frac{\langle j(j-1)\rangle r_{f}\left(a+D B_{n}{ }^{2}\right) \psi_{n}(\mathbf{x}) \psi_{m}\left(\mathbf{x}^{\prime}\right)}{\left(2 a+D B_{n}{ }^{2}+D B_{m}{ }^{2}\right)\left[\left(a+D B_{n}{ }^{2}\right)^{2}+\omega^{2}\right.}\right] \\
& \quad+r_{D}(\mathbf{x}) r_{D}\left(\mathbf{x}^{\prime}\right) \sum_{i, n, m=1}^{\infty} C_{i n m} A_{i}{ }^{N} \frac{\langle j(j-1)\rangle r_{f}\left(a+D B_{m}{ }^{2}\right) \psi_{n}\left(\mathbf{x}^{\prime}\right) \psi_{m}(\mathbf{x})}{\left(2 a+D B_{n}{ }^{2}+D B_{m}{ }^{2}\right)\left[\left(a+D B_{m}\right)^{2}+\omega^{2}\right]}
\end{aligned}
$$

To analyse specifically Ricker's P.S.D. experiment we represent his reactor as a cube, and generate the various reactor parameters from a measured $\beta / \Lambda$ (fraction of delayed neutrons over neutron lifetime in units of $k$, the multiplication constant) and a representative $L$ (diffusion length) for his reactor. Of course (85) has been derived ignoring delayed neutrons; however, if we include a single group of delayed neutrons, and then keep only terms of zero order in the small quantity $\lambda / r_{a}$ ( $\lambda$ being the single precursor group time constant) we obtain in the frequency range of experimental interest $(\lambda \ll \omega<b)$ an identical expression for $\Phi\left(\mathbf{x}, \mathbf{x}^{\prime}, \omega\right)$ where the critical condition is now $a-\langle\zeta\rangle r_{f}+D B_{1}^{2}-0(\zeta \zeta\rangle$ being the mean number of delayed neutrons produced per fission). This result is demonstrated in Appendix A.

In Fig. 3 a comparison is made between Ricker's measurement of the P.S.D. for his system in the critical limit and the corresponding result obtained from $\Phi\left(\mathbf{x}, \mathbf{x}^{\prime}, \omega\right)$ integrated over an appropriate detector (one which resembles the actual detector as closely as possible). The configuration of the detector-reactor system is also illustrated in Fig. 3. The comparison is clearly not very satisfactory. Our expression for theP.S.D. does not fit Ricker's data, and no small variations of the reactor parameters will make it do so. A similar result has been obtained by Sheff for a reactor much the same as that treated here, but for a point detector located at approximately the centre of our finite detector. His result is equally unsatisfactory as far as explaining this experiment is concerned.

It is important to note for future reference, that in order to compute $\Phi(\omega)$ with sufficient accuracy, it is necessary to keep 64 terms in the modal expansion of equation (85). This is the direct result of the smallness of the ratio $L / R$ where $R$ is a characteristic reactor dimension, and the fact that the leakage rate is proportional to the square of this ratio.

Again a change in our reactor model is called for. An experiment which was analysed, with what has appeared to be great success, by the simplest of theories seems to demand more and more detail from a more general theory. 


\section{FINITE REACTOR MODEL-FAST LEAKAGE INCLUDED}

We show here that the difficulties encountered with the reactor model of the preceding section disappear when fast diffusion and leakage are taken into account. It is well known that the picture of neutrons thermalizing at a point (implicit in the treatment of Section 4), is unrealistic. In fact, in the small, water-moderated reactors used by Ricker and Albrecht, fast leakage is the primary contribution to the leakage from the core. Moreover, as we shall show, it turns out that a reasonable treatment of fast diffusion is crucial to the understanding of reactor fluctuation experiments. When fast diffusion is taken into account we not only find immediate agreement between our calculations and the experimental results, but we are also able to arrive at an explanation for the practical success of the space-independent theory in treating correlation problems where space effects, by definition, should play an important role.

First, we sketch a generalization of the treatment of Section 4, and apply it to a two-group problem. The details of this are shown in Appendix B. Along with the neutron doublet density $N^{v x}$ which we now take to mean thermal neutron doublet density, we now work with $N^{v_{n}}, N^{n x}, N^{n n}$, the thermal-fast, fast-thermal and fast neutron doublet densities respectively. In place of equation (80) one obtains four coupled equations for the corresponding correlation functions, and is hence led to a coupled set of equations for expansion coefficients $A_{n m}{ }^{N N}, A_{n m}{ }^{n N}, A_{m n}{ }^{n N}$ and $A_{n m}{ }^{n n}$. An approximate solution for the thermal neutron doublet expansion coefficient is obtained to lowest order in the small parameter $r_{a} / r_{R},\left(r_{R}\right.$ being the removal rate from the fast group, see Appendix B), with the following conclusions:

(i) The resulting expression for the P.S.D. is similar in form to the one-group result with extra factors of $\left(1+\tau B_{n}^{2}\right)^{-1}$ appearing in various places. These serve to depress the effect of higher mode contributions to the P.S.D. In fact, for the reactor used by Ricker, the two-group treatment has the effect of collapsing the infinite sum in (85) to a single term, $n=m=1$, whereas before it was necessary to keep approximately 64 terms to represent $\Phi(\omega)$ adequately.

(ii) For the surviving term, the two-group result may be obtained directly from the lowest mode contribution to equation (85) by a transcription which simply replaces the diffusion length $L$ by $M$, the migration length, where

$$
M^{2}=L^{2}+\tau, \tau=\text { neutron age to thermal. }
$$

This can also be seen from the Fermi age treatment of thermalization utilized in Raievski's inductive theory of neutron noise.

In Fig. 4, the single term expression for P.S.D. with $M$ replacing $L$ is compared with Ricker's experimental results, and the agreement with the measured spectrum (the solid squares) is excellent. To facilitate comparison the first term approximation of $\Phi(\omega)$ is presented in a notation analogous to that used by Ricker. The dependence on the delayed neutron precursor decay constant $\lambda$ is left explicit. The details of this lowest mode calculation are also found in Appendix A. For a subcritical reactor (with external sources distributed in the lowest mode)

$$
\left(\frac{2}{\Gamma^{2}}\right) \Phi(\omega)-r_{D} \overline{N^{N}}+r_{D}{ }^{2} \overline{N^{N}} \bar{C} \frac{\langle j(j-1)\rangle}{\Lambda\langle\nu\rangle}\left\{\frac{\left(\Lambda / \beta^{2}\right)}{\left(\frac{\omega^{2}}{\omega^{2}+\lambda^{2}}-\rho\right)^{2}+\omega^{2}\left(\frac{\Lambda}{\beta}+\frac{\lambda}{\omega^{2}+\lambda^{2}}\right)^{2}}\right\}
$$




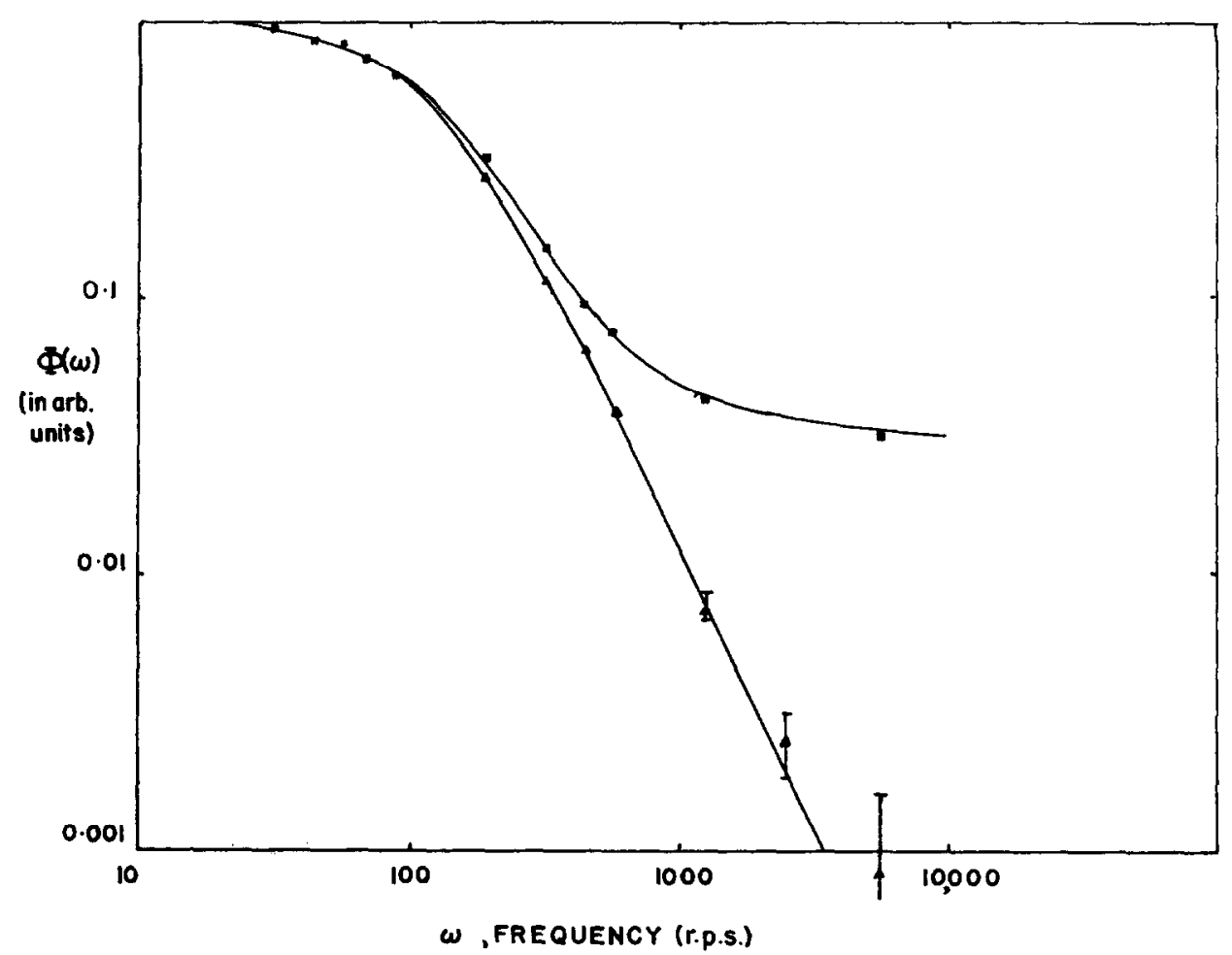

FIG. 4.--Measured and calculated P.S.D. for the same system considered in Fig. 3. Where now the calculations are performed from the first term in the sum (85) with $D=$ $r_{a} M^{2}, M=7.06 \mathrm{~cm}$ (the migration length).

and for a critical reactor

$$
\left(\frac{2}{\Gamma^{2}}\right) \Phi(\omega)=r_{D} \overline{N^{N}}+r_{D}{ }^{2} \overline{N^{N}} \bar{C} \frac{\langle j(j-1)\rangle}{\Lambda\langle\boldsymbol{v}\rangle}\left\{\frac{(\Lambda / \beta)^{2}}{\left(\frac{\omega^{2}}{\omega^{2}+\lambda^{2}}\right)^{2}+\omega^{2}\left(\frac{\Lambda}{\beta}+\frac{\lambda}{\omega^{2}+\lambda^{2}}\right)^{2}}\right\}
$$

where

$$
\overline{N^{N}} \equiv \int_{\text {D.V. }} \mathrm{d}^{3} \mathbf{x} A_{1}{ }^{N} \psi_{1}(\mathbf{x})
$$

is the mean neutron population in the detector,

$$
\bar{C} \equiv \int_{\text {D.V. }} \mathrm{d}^{3} \mathbf{x} C_{111} \psi_{1}(\mathbf{x})
$$

is the importance factor for detector geometry and configuration,

$$
\langle\boldsymbol{\nu}\rangle \equiv\langle j\rangle+\langle\zeta\rangle
$$

is the total mean neutron production per fission, and

is the reactivity in units of $\beta$.

$$
\rho \equiv \equiv \frac{\langle\nu\rangle r_{f}-r_{a}\left(1+M^{2} B_{1}^{2}\right)}{\langle\zeta\rangle r_{f}}
$$


Comparing these expressions with those used by Ricker (p. 67) we can see that each corresponding expression has precisely the same form.

It is now possible to give an explanation for the practical success of the space and energy-independent theory. The manner in which a detector samples the density of correlated neutrons may, in general, play a role in determining the P.S.D. For a given detector, the P.S.D. depends upon the spatial dependence of the doublet density and therefore on reactor configuration, neutron energy distribution, and degrec of multiplication. However, in a reactor such as that used by Ricker et al., the ratio of the migration length to the greatest linear dimension is large enough to render the nonsingular part of the doublet density a factorizable function of its two spatial variables. The spatial dependence is given by the product, $\psi_{1}(\mathbf{x}) \psi_{1}\left(\mathbf{x}^{\prime}\right)$, of the lowest eigenfunctions of the Boltzmann operator. Different detectors sampling such a density may yield different results for the overall magnitudes of the P.S.D., but the characteristic Lorentzian frequency dependence will be the same in each case. This dependence is fixed by the approximate factorizability of the doublet density as a product of eigenfunctions of the appropriate Boltzmann operator. Thus since the space and energyindependent theory doublet density is trivially factorizable in this sense, the success of this theory in analysing Ricker's experiment is guaranteed. Of course the full space and energy-dependent analysis is necessary to interpret the details even of these experiments. The relative magnitudes of the plateaus in the P.S.D. (see Fig. 4) are determined by $\bar{C}$, the detector configuration importance factor, and $r_{D}$, the detection rate.

$\vec{C}$ does not arise in the space and energy-independent theory, and the plateaus in the measured data must be fitted by choosing a value for $r_{D}{ }^{*}$ which is interpreted to be an effective detection rate for the real detector smeared out over the reactor volume. Finally the overall magnitude of the P.S.D. is seen in the general treatment to be proportional to $\overline{N^{N}}$, the expected number of neutrons in the detector volume; whereas for the space and energy-independent analysis the proportionality is with the average neutron density (number $/ \mathrm{cm}^{3}$ ) for the reactor. But as there has been little practical interest in the absolute magnitude of the P.S.D., this discrepancy has not hindered the interpretation of experiments.

The explanation above also applies to the success of the space and energy-independent theory in interpreting Albrecht's variance-to-mean experiment. From equation (84) our general expression for the detected particle variance follows immediately:

$$
\begin{aligned}
\mathrm{X}^{D D}\left(\mathbf{x}, \mathbf{x}^{\prime}, t\right)= & r_{D}(\mathbf{x}) \delta\left(\mathbf{x}-\mathbf{x}^{\prime}\right) N^{N}(\mathbf{x}) t+2 r_{D}\left(\mathbf{x}^{\prime}\right) r_{D}\left(\mathbf{x}^{\prime}\right)\langle j(j-1)\rangle r_{f} \\
& \times \sum_{i, n, m=1}^{\infty} C_{i n m} A_{i}^{N} \frac{\psi_{n}(\mathbf{x}) \psi_{m}\left(\mathbf{x}^{\prime}\right) t}{\left[2 a+D B_{n}{ }^{2}+D B_{m}{ }^{2}\right]\left[a+D B_{n}{ }^{2}\right]} \\
& \times\left\{1-\frac{1-\mathrm{e}^{-\left(a+D B_{n}{ }^{2}\right) \eta}}{\left(a+D B_{n}{ }^{2}\right) t}\right\}
\end{aligned}
$$

Again, taking fast diffusion into account, for a reactor small enough and near enough to critical, this sum is well represented by its first term with $D$ set equal to $r_{a} M^{2}$. The

\footnotetext{
* We also used $r_{D}$ as a fitting parameter, for as $\bar{C}$ depends critically on the extrapolated boundaries and detector geometry chosen to represent the system, we would not expect our calculated P.S.D. to fit the relative plateau heights as measured by Ricker. However, the particular value of $r_{D}(8 \cdot 35 \times$ $10^{3} \mathrm{sec}^{-1}$ ) chosen to effect a fit, is of the correct order-of-magnitude for the type of detector actually used: and we are, therefore, confident that the calculated $\bar{C}$ (and thus our reactor-detector configuration) is not unrealistic.
} 
result for the variance-to-mean follows immediately and is seen to be of the same form as that obtained from the space and energy-independent theory used by Albrecht, i.e.

$$
V / M=1+\bar{\epsilon} \frac{\langle j(j+1)\rangle}{\langle\nu\rangle \beta[1+K(1-\beta)][1-\rho]}\left\{1-\frac{1-\exp \{-[\beta(1-\rho) / \Lambda] t\}}{[\beta(1-\rho) / \Lambda] t}\right),
$$

where

$$
\bar{\epsilon} \equiv \frac{r_{D}}{r_{a}+D B_{1}^{2}} \int_{\text {D.V. }} \mathrm{d}^{3} \mathbf{x} C_{111} \psi_{1}(\mathbf{x})=\frac{r_{D} \bar{C}}{r_{a}+D B_{1}{ }^{2}}
$$

is the detector efficiency, and

is the multiplication factor.

$$
K \equiv\langle\boldsymbol{v}\rangle r_{f} /\left(r_{a}+D B_{1}{ }^{2}\right)
$$

It is interesting to note that $\bar{C}$ can be written in the following form:

where

$$
\bar{C}=\frac{V_{D}}{V_{R}} \Phi
$$

and

$$
\begin{aligned}
V_{D}= & \text { detector volume, } \\
V_{R}= & \text { reactor volume, } \\
\bar{\phi}= & \text { the appropriately weighted (by } C_{111} \text { ) average of the lowest } \\
& \text { mode over the detector volume. }
\end{aligned}
$$

We are thus in agreement with the interpretation of detector efficiency commonly used in conjunction with the space and energy-independent theory (i.e. $\bar{\epsilon}$ being proportional to the ratio of detection rate to total neutron loss rate in the reactor).

We therefore conclude that the success of the space and energy-independent theory in the analysis of the experiments considered here is limited to the class of reactors in which the migration length is large enough with respect to the overall size. The choice of a different reactor (one in which $M / R \leqslant 1$ ) might give results which such a theory could not explain. To support this claim, we have displayed in Fig. 5 results calculated for the P.S.D. in a large graphite-moderated reactor (the XP-10 at Oak Ridge*) with a variety of detector configurations and locations. Specifically we consider a 'point-like' detector (represented by a single $\mathrm{BF}_{3}$ tube) and 'line-like' detector (represented by three disjoint $\mathrm{BF}_{3}$ tubes feeding a single output.) Calculations of the P.S.D. are made for these detectors located first at the centre of the reactor and then off-centre (see Fig. 5) where higher reactor modes have more influence. The 'characteristic behaviour' of these P.S.D.s $\left(\left(2 / \Gamma^{2}\right) \Phi(\omega)-r_{D^{N}}\right)$ is plotted, and the $\omega^{-2}$ behaviour, characteristic of the space and energy-independent theory, definitely does not appear.

It should be pointed out that P.S.D. measurements in such a system may be experimentally difficult to achieve. If $r_{D}$ and/or $V_{D}$ cannot be made sufficiently great, the constant term in the P.S.D. will swamp all frequency dependence. The detector used by Ricker et al. would not be satisfactory. Perhaps 'cross' P.S.D. measurements, in which no constant term appears, are better suited for such reactors.

* The authors wish to thank Professor S. H. Hanauer for pointing out the experimental possibilities for XP-10, and for additional valuable discussions on the nature of the reactor on which he, Ricker and Mann performed their P.S.D. experiments. 


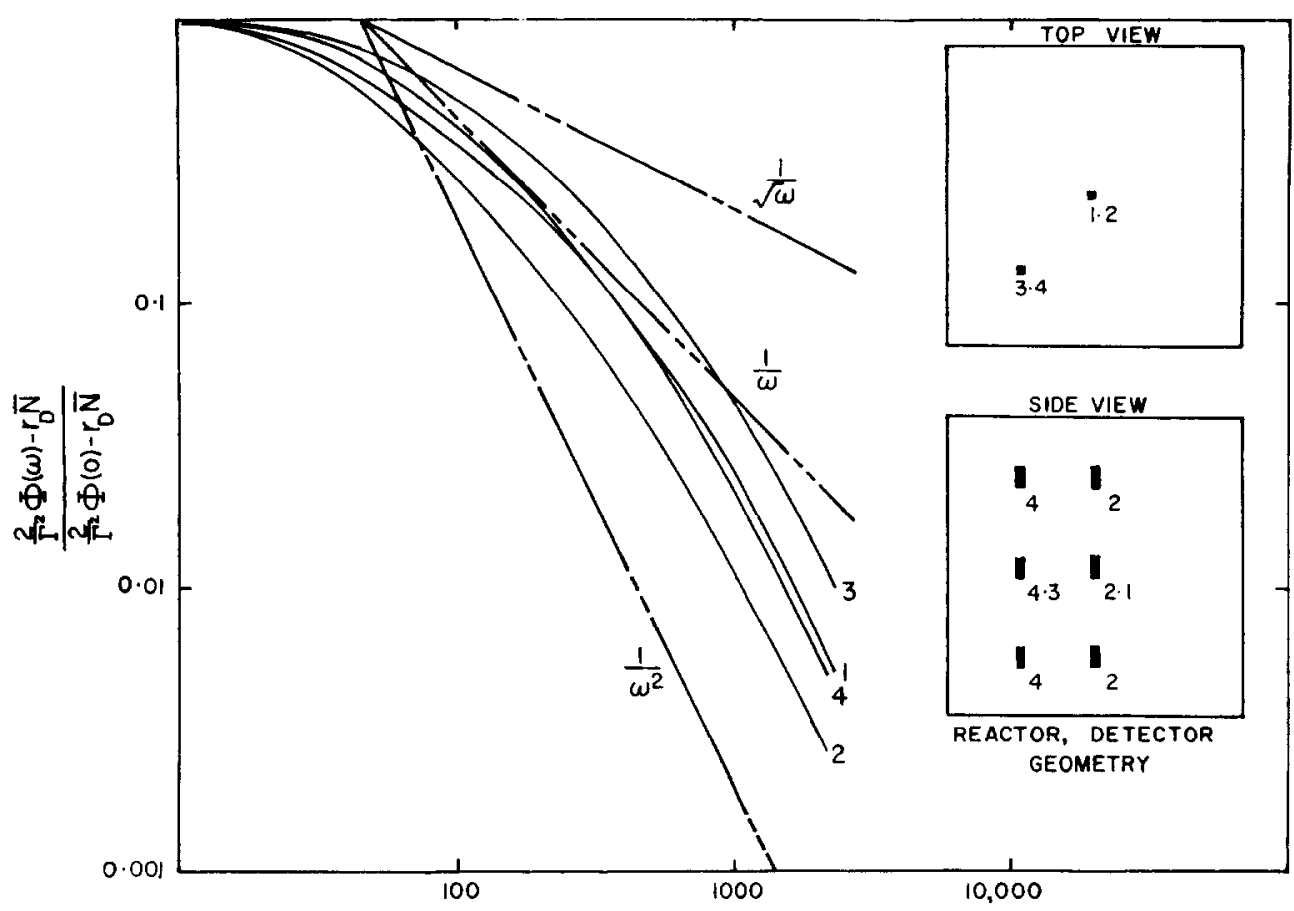

$\omega$, FREQUENCY (r.p.s.)

FIG. 5.- Characteristic frequency dependence of the P.S.D. as calculated from equation (B.24) for various detector configurations in a large graphite-moderated reactor $(\beta / \Lambda=$ $7 \mathrm{sec}^{-1}, r_{a}=1000 \mathrm{sec}^{-1} k_{\infty}=1.035$ and $M^{2}=560$ ). The reactor is a bare homogenous cube. Curve 1 represents the frequency dependence for a single $2 \times 2 \mathrm{~cm} \times 30 \mathrm{~cm}$ rectangular parallel-piped detector located at the centre of the reactor. Curve 3 is for a similar detector located $250 \mathrm{~cm}$ from the bottom of the reactor and $125 \mathrm{~cm}$ in from two adjacent sides. Curve 2 is for a three segment detector whose middle segment corresponds to the single detector of Curve 1 . Curve 4 is for a similar three segment detector whose middle segment corresponds to the single detector of Curve 3.

As a final notc on Fig. 5, it is interesting to observe that for detectors placed in regions of the reactor where the higher modes have greatest importance, the reactor seems to appear infinite. The resulting P.S.D.s are very much like those predicted in the infinite reactor, finite detector model (see Table 1). It should also be pointed out that when fast diffusion is taken into account the infinite reactor results of Section 3 are modified (in the frequency range of interest $a \approx \omega<r_{a}<r_{R}$ ) merely by the replacement of $L^{2}$ by $M^{2}$ in the diffusion coefficient. The calculation is shown in Appendix C. This emphasizes the non-trivial nature of the interaction between detector configuration, reactor size and the distribution of correlated neutrons in P.S.D. measurements. For a given detector configuration the inclusion of fast diffusion takes the calculated P.S.D. farther away from a Lorentzian shape for an infinite reactor and closer to it for a finite reactor.

Acknowledgment -We wish to express our gratitude to Professor ZrYa Akcasu for many stimulating discussions and helpful suggestions.

AKCASU Z. To be published.

\section{REFERENCES}

Albrecht R. W. (1962) Nucl. Sci. Engng 14, 153.

Bennett E. F. (1960) Nucl. Sci. Engng 8, 56. 
HARRIS D. H. (1965) Nucl. Sci. Engng 21, 369.

MATTHF. W. (1962) Nukleonik 4, 213.

Osborn R. K. (1965) Report ORNL-3757.

Osborn R. K. and AKCasu Z. (1965) Neutron Dynamics and Control, AEC Symposium Series.

Osborn R. K. and NAtelson M. (1965) J. nucl. Energy (Parts A/B Reactor Sci. Technol.) 19, 169.

OSBORN R. K. and YIP S. (1963) The Foundations of Neutron Transport Theory.

Pluta P. R. (1961) University of Michigan, Industry Programme Report, No. 569.

RAIEVSKi V. (1960) Report CEA 1973.

Ricker C. W., Hanauer S. H. and Mann (1965) E. R. Report ORNL-TM-1066.

SHefF J. R. (1965) The Cross Correlation of the Neutron Density at Two Points in a Nuclear Reactor, Ph.D. Thesis, University of Washington.

Sheff J. R. and Albrecht R. W. (1964) Trans. Am. nucl. Soc. 7, (1) 61.

\section{APPENDIX A}

Here we shall include the statistical behaviour of a single group of delayed neutron precursors. It turns out that the expression (85) for the generalized P.S.D. is not affected by this, at least to lowest order in the small parameter $\lambda / r_{a} \equiv \epsilon$ where $\lambda$ is the precursor decay constant.

We use the superscript $A$ to refer to precursor quantities. The basic set of equations (see Section 2) is generalized as follows:

$$
\begin{aligned}
\frac{\partial}{\partial t} N^{D}(\mathbf{x}, t) & =r_{D}(\mathbf{x}) H(t) N^{N}(\mathbf{x}), \\
{\left[a-D \nabla^{2}\right] N^{N}(\mathbf{x}) } & -\lambda N^{A}(\mathbf{x})+S(\mathbf{x}), \\
\lambda N^{A}(\mathbf{x}) & =\langle\zeta\rangle r_{j} N^{N}(\mathbf{x}),
\end{aligned}
$$

where $\langle\zeta\rangle$ is the expected number of precursors produced per fission

$$
\begin{aligned}
{\left[\frac{\partial}{\partial t}+a-D \nabla^{2}\right] \mathrm{X}^{N D}\left(\mathbf{x}, \mathbf{x}^{\prime}, t\right)=} & r_{D}\left(\mathbf{x}^{\prime}\right) H(t) \mathrm{X}^{N N}\left(\mathbf{x}, \mathbf{x}^{\prime}\right)+\lambda \mathrm{X}^{A D}\left(\mathbf{x}, \mathbf{x}^{\prime}, t\right) \\
& -r_{D}\left(\mathbf{x}^{\prime}\right) H(t) \delta\left(\mathbf{x}-\mathbf{x}^{\prime}\right) N^{N}(\mathbf{x}) \\
{\left[\frac{\partial}{\partial t}+\lambda\right] \mathrm{X}^{A D}\left(\mathbf{x}, \mathbf{x}^{\prime}, t\right)=} & r_{D}\left(\mathbf{x}^{\prime}\right) H(t) \mathrm{X}^{N A}\left(\mathbf{x}, \mathbf{x}^{\prime}\right)+\langle\zeta\rangle r_{f} \mathrm{X}^{N D}\left(\mathbf{x}, \mathbf{x}^{\prime}, t\right) \\
{\left[a-D \nabla^{2}+\lambda\right] \mathrm{X}^{N A}\left(\mathbf{x}, \mathbf{x}^{\prime}\right)=} & \langle\zeta\rangle r_{r} \mathrm{X}^{N N}\left(\mathbf{x}, \mathbf{x}^{\prime}\right)+\lambda \mathrm{X}^{A A}\left(\mathbf{x}, \mathbf{x}^{\prime}\right) \\
& -\delta\left(\mathbf{x}-\mathbf{x}^{\prime}\right)\left[\lambda N^{A}(\mathbf{x})+\langle\zeta\rangle r_{f} N^{N}(\mathbf{x})-\langle j\rangle\langle\zeta\rangle r_{f} N^{N}(\mathbf{x})\right] \\
{\left[2 a-D \nabla^{2}-D \nabla^{\prime 2}\right] \mathrm{X}^{N N}\left(\mathbf{x}, \mathbf{x}^{\prime}\right)=} & \lambda \mathrm{X}^{N A}\left(\mathbf{x}, \mathbf{x}^{\prime}\right)+\lambda \mathbf{X}^{N A}\left(\mathbf{x}^{\prime}, \mathbf{x}\right) \\
& +\delta\left(\mathbf{x}-\mathbf{x}^{\prime}\right)\left[C N^{N}(\mathbf{x})+\lambda N^{A}\left(\mathbf{x}^{\prime}\right)+S(\mathbf{x})\right] \\
& +2 \nabla \cdot \nabla^{\prime} \delta\left(\mathbf{x}-\mathbf{x}^{\prime}\right) N^{N}(\mathbf{x}) \\
2 \lambda \mathrm{X}^{A A}\left(\mathbf{x}, \mathbf{x}^{\prime}\right)= & \langle\zeta\rangle r_{f} \mathrm{X}^{N A}\left(\mathbf{x}, \mathbf{x}^{\prime}\right)+\langle\zeta\rangle r_{f} \mathrm{X}^{N A}\left(\mathbf{x}^{\prime}, \mathbf{x}\right) \\
& \left.+\delta\left(\mathbf{x}-\mathbf{x}^{\prime}\right)[\zeta\rangle r_{f} N^{N}(\mathbf{x})+\lambda N^{A}(\mathbf{x})\right] \\
\frac{\partial}{\partial t} \mathrm{X}^{D D}\left(\mathbf{x}, \mathbf{x}^{\prime}, t\right)= & r_{D}(\mathbf{x}) H(t) \mathrm{X}^{N D}\left(\mathbf{x}, \mathbf{x}^{\prime}, t\right)+r_{D}\left(\mathbf{x}^{\prime}\right) H(t) \mathrm{X}^{N D}\left(\mathbf{x}^{\prime}, \mathbf{x}, t\right) \\
& +r_{D}(\mathbf{x}) H(t) \delta\left(\mathbf{x}-\mathbf{x}^{\prime}\right) N^{N}(\mathbf{x})
\end{aligned}
$$

The reactor is considered to be in the steady state, and the approximation scheme discussed in Section 3 has been applied.

Using the eigenfunction expansion of equation (78), we are led to the following set of coupled equations for the expansion coefficients of $\mathrm{X}^{N N}\left(\mathbf{x}, \mathbf{x}^{\prime}\right), \mathrm{X}^{N A}\left(\mathbf{x}, \mathbf{x}^{\prime}\right), \mathrm{X}^{N A}\left(\mathbf{x}^{\prime}, \mathbf{x}\right)$, and $\mathrm{X}^{A A}\left(\mathbf{x}, \mathbf{x}^{\prime}\right)$ :

$$
\begin{aligned}
{\left[2 a+D B_{n}{ }^{2}+D B_{m}{ }^{2}\right] A_{n m}{ }^{N N}-\lambda A_{n m}{ }^{N A}-\lambda A_{m m}{ }^{N A} } & =\sum_{i=1}^{\infty} C_{i n m} A_{i}{ }^{N}\left[C+a+D B_{n}{ }^{2}+D B_{m}{ }^{2}\right] \\
-\langle\zeta\rangle r_{f} A_{n m}{ }^{N N}+\left[a+D B_{n}{ }^{2}+\lambda\right] A_{n m}{ }^{N A}-\lambda A_{n m}{ }^{A A} & =-\sum_{i=1}^{\infty} C_{i n m} A_{i}{ }^{N}\langle\zeta\rangle r_{f}[\langle j\rangle-2] \\
-\langle\zeta\rangle r_{f} A_{n m}{ }^{N N}+\left[a+D B_{m}{ }^{2}+\lambda\right] A_{m n}{ }^{N A}-\lambda A_{n m}{ }^{A A} & =\sum_{i=1}^{\infty} C_{i n m} A_{i}{ }^{N}\langle\zeta\rangle r_{f}[\langle j\rangle \cdots 2] \\
-\langle\zeta\rangle r_{f} A_{n m}{ }^{N A}-\langle\zeta\rangle r_{f} A_{m n}{ }^{N A}+2 \lambda A_{n m}{ }^{A A} & =2 \sum_{i=1}^{\infty} C_{i n m} A_{i}{ }^{N}\langle\zeta\rangle r_{f}
\end{aligned}
$$


with the solution:

$$
\begin{aligned}
A_{n m}{ }^{N \nu}=\sum_{i=1}^{\infty} C_{i n m} A_{i}{ }^{j}\left\{\left[C+a+D B_{n}{ }^{2}\right.\right. & \left.+D B_{m}{ }^{2}\right] \Delta A \\
& \left.+\langle\zeta\rangle r_{f}[\langle j\rangle-2](\Delta B-\Delta C)-2\langle\zeta\rangle r_{f} \Delta D\right\} / \Delta M
\end{aligned}
$$

where

$$
\Delta M=\left|\begin{array}{cccr}
2 a+D B_{n}{ }^{2}+D B_{m}{ }^{2} & -\lambda & -\lambda & 0 \\
-\langle\zeta\rangle r_{f} & a+D B_{n}{ }^{2}+\lambda & 0 & -\lambda \\
-\langle\zeta\rangle r_{f} & 0 & a+D B_{m}{ }^{2}+\lambda & -\lambda \\
0 & -\langle\zeta\rangle r_{f} & -\langle\zeta\rangle r_{f} & 2 \lambda
\end{array}\right|=\left[\begin{array}{c}
\left.2 a+D B_{n}{ }^{2}+D B_{m}{ }^{2}\right] \Delta A \\
+\langle\zeta\rangle r_{f}(\Delta B-\Delta C),
\end{array}\right.
$$

and where $\Delta A, \Delta B, \Delta C$ and $\Delta D$ are the minors of the elements of the first column of $\Delta M$. Similarly, the solution for $A_{n m}{ }^{N A}$ is:

$$
\begin{aligned}
A_{n m}{ }^{N A}=\sum_{i=1}^{\infty} C_{i n m} A_{i}{ }^{\nu}\left\{-\left[C+a+D B_{n}{ }^{2}+\right.\right. & \left.D B_{m}{ }^{2}\right] \Delta E \\
& \left.+\langle\zeta\rangle r_{f}[\langle j\rangle-2](\Delta F-\Delta G)+2\langle\zeta\rangle r_{j} \Delta H\right\} / \Delta M
\end{aligned}
$$

where $\Delta E, \Delta F, \Delta G$ and $\Delta H$ are the minors of the elements of the second column of $\Delta M$.

For the generalized P.S.D. we need only examine the expression for $A_{n}{ }^{N D}\left(\mathbf{x}^{\prime}, s\right)$, which is obtained by Laplace transforming and combining equations (A.4) and (A.5):

$$
\begin{aligned}
& {\left[s^{2}+\left(a+D B_{n}{ }^{2}+\lambda\right) s+\lambda\left(a+D B_{n}{ }^{2}-\langle\zeta\rangle r_{f}\right)\right] A_{n}{ }^{N A}\left(\mathbf{x}^{\prime}, s\right)} \\
& \quad=r_{D}\left(\mathbf{x}^{\prime}\right) \sum_{m=1}^{\infty}\left\{A_{n m}{ }^{N N}-\sum_{i=1}^{\infty} C_{i n m} A_{i}{ }^{N}\right\} \frac{(s+\lambda)}{\lambda} \psi_{m}\left(\mathbf{x}^{\prime}\right)+r_{D}\left(\mathbf{x}^{\prime}\right) \sum_{m=1}^{\infty} A_{n m}{ }^{N D} \frac{\lambda}{s} \psi_{m}\left(\mathbf{x}^{\prime}\right) .
\end{aligned}
$$

From equation (A.17) and the definition of the P.S.D. (3) the following expansion in reactor modes is found for the P.S.D.

$$
\begin{aligned}
\left(\frac{2}{\Gamma^{2}}\right) \Phi(\omega, & \left.\mathbf{x}, \mathbf{x}^{\prime}\right)-r_{D}(\mathbf{x}) \delta\left(\mathbf{x}-\mathbf{x}^{\prime}\right) N^{N}(\mathbf{x})+r_{D}(\mathbf{x}) r_{D}\left(\mathbf{x}^{\prime}\right) \sum_{n, m=1}^{\infty} \psi_{n}(\mathbf{x}) \psi_{m}\left(\mathbf{x}^{\prime}\right) \\
& \times\left\{\left(\frac{\omega^{2}\left(a+D B_{n}{ }^{2}\right)+\lambda^{2}\left(a+D B_{n}{ }^{2}-\langle\zeta\rangle r_{j}\right)}{\left.\left(\lambda\left(a+D B_{n}{ }^{2}-\langle\zeta\rangle r_{f}\right)-\omega^{2}\right)^{2}+\omega^{2}(a) D B_{n}{ }^{2}+\lambda\right)^{2}}\right)\left[A_{n m}{ }^{N N}-\sum_{i=1}^{\infty} C_{i n n n} A_{i}{ }^{N}\right]\right. \\
& \left.+\left(\frac{\lambda^{2}\left(a+D B_{n}{ }^{2}-\langle\zeta\rangle r_{f}\right)-\lambda \omega^{2}}{\left(\lambda\left(a+D B_{n}{ }^{2}-\langle\zeta\rangle r_{f}\right)-\omega^{2}\right)^{2}+\omega^{2}\left(a+D B_{n}{ }^{2}+\lambda\right)^{2}}\right) A_{n m}{ }^{N A}\right\} \\
& +r_{D}(\mathbf{x}) r_{D}\left(\mathbf{x}^{\prime}\right) \sum_{n, m=1}^{\infty} \psi_{n}\left(\mathbf{x}^{\prime}\right) \psi_{m}(\mathbf{x})\{n \rightleftarrows m\} .
\end{aligned}
$$

In the frequency range of experimental interest $\omega \gg \lambda$, thus the quantities appearing in (A.18) can be obtained to lowest order in $\epsilon$ simply by setting the rate $\lambda=0$ wherever it appears, except when it is to be compared with the rate $a+D B_{1}{ }^{2}-\langle\zeta\rangle r_{f}$ which becomes small near criticality, or perhaps when it multiplies the rate $a+D B_{n}{ }^{2}$ which can be large for large $n$. We therefore consider the term $n=m=1$ in the double sum (A.18) separately from the others.

For the case $n=m=1$, we combine the exact expressions (A.10-A.13) with $n=m=1$ to obtain the lowest mode contribution to the P.S.D.

$$
\begin{aligned}
& \left(\frac{2}{\Gamma^{2}}\right) \Phi_{11}\left(\mathbf{x}, \mathbf{x}^{\prime}, \omega\right)=r_{D}(\mathbf{x}) \delta\left(\mathbf{x}-\mathbf{x}^{\prime}\right) N^{N}(\mathbf{x}) \\
& \quad+r_{D}(\mathbf{x}) r_{D}\left(\mathbf{x}^{\prime}\right)\langle j(j-1)\rangle r_{f} \sum_{i=1}^{\infty} \frac{C_{i 11} A_{i} \psi_{1}(\mathbf{x}) \psi_{1}\left(\mathbf{x}^{\prime}\right)\left(\omega^{2}+\lambda^{2}[1+\langle j\rangle\langle\zeta\rangle\langle j(j-1)\rangle]\right)}{\left(\lambda\left(a+D B_{1}{ }^{2}-\langle\zeta\rangle r_{j}\right)-\omega^{2}\right)^{2}+\omega^{2}\left(a+D B_{1}{ }^{2}+\lambda\right)^{2}} .
\end{aligned}
$$

The constant term has been included to emphasize the comparison with Ricker's results (p. 27). Indeed Ricker's expression, with slight notational changes is identical with (A.19) with the small term $\langle j\rangle\langle\zeta\rangle\langle j(j-1)\rangle$ neglected.

We note that there is no difficulty in taking the limits $\lambda \rightarrow 0$ and $a+D B_{1}{ }^{2}-\langle\zeta\rangle r_{f} \rightarrow 0$ (critical limit) separately, or together in either order. 
In the case when either $n$ or $m$ is greater than 1 , we perform the asymptotic analysis directly on the expression (A.18) for the P.S.D. and the solutions (A.14-A.16) for the $A_{n m}$ 's. The frequencydependent coefficients in the double sum (A.18) both possess the same denominator. To lowest order in $\lambda / \omega$, the term $\lambda\left(a+D B_{n}{ }^{2}-\langle\zeta\rangle r_{f}\right)$ may be ignored regardless of the value of $n$, since for $\omega \ll a+$ $D B_{n}^{2}$ we have

$$
\lambda\left(a+D B_{n}^{2}-\langle\zeta\rangle r_{f}\right)-\omega^{2} \ll \omega\left(a+D B_{n}^{2}+\lambda\right),
$$

whereas if $\omega$ is at least the same order of magnitude as $a+D B_{n}{ }^{2}$ we have

$$
\lambda\left(a+D B_{n}^{2}-\langle\zeta\rangle r_{\gamma}\right) \ll \omega^{2} .
$$

Thus the denominator becomes simply

$$
\text { Denominator } \approx \omega^{2}\left(\omega^{2}+\left(a+D B_{n}^{2}\right)^{2}\right) .
$$

We have also made use of the fact that $\lambda \ll\langle\zeta\rangle r_{f}$.

The determinental solutions for (A.14), (A.16) for the $A_{n m}$ 's may also be easily evaluated to lowest order in $\epsilon$. We find

$$
\frac{\Delta B}{\Delta M} \approx \frac{\Delta C}{\Delta M} \approx \frac{\Delta D}{\Delta M} \approx \frac{\Delta B}{\Delta A} \approx \frac{\Delta C}{\Delta A} \approx 0 \text { and } \frac{\Delta A}{\Delta M} \approx \frac{1}{\left(2 a+D B_{n}{ }^{2}+D B_{m}{ }^{2}\right)} .
$$

Thus to lowest order in $\epsilon$

$$
A_{n m}{ }^{N N}=\sum_{i=1}^{\infty} C_{i n m} A_{i}{ }^{N}\left\{1+\frac{\langle j(j-1)\rangle r_{f}}{\left(2 a+D B_{n}^{2}+D B_{m}{ }^{2}\right)}\right\} .
$$

$A_{n m}^{N A}$ may also be computed to lowest order in $\epsilon$ but this is not necessary, since the frequencydependent coefficient multiplying it in (A.18) is higher order in $\epsilon$ than the coefficient multiplying the $\operatorname{term}\left(A_{n m}{ }^{N N}-\sum_{i=1}^{\infty} C_{i n m} A_{i}{ }^{N}\right)$.

Finally, to lowest order in $\epsilon$, the coefficient of the term $\left(A_{n m}{ }^{N N}-\sum_{i=1}^{\infty} C_{i n m} A_{i}{ }^{N}\right)$ can be written as

$$
\left(\frac{\omega^{2}\left(a+D B_{n}{ }^{2}\right)+\lambda^{2}\left(a+D B_{n}{ }^{2}-\langle\zeta\rangle r_{f}\right)}{\left(\lambda\left(a+D B_{n}{ }^{2}-\langle\zeta\rangle r_{f}\right)-\omega^{2}\right)^{2}+\omega^{2}\left(a+D B_{n}{ }^{2}+\lambda\right)^{2}}\right) \approx \frac{a+D B_{n}{ }^{2}}{\omega^{2}+\left(a+D B_{n}{ }^{2}\right)^{2}},
$$

and combining (A.24), (A.18) [with $\lambda=0$ ] and the first term result (A.19) we have the desired expression for the generalized P.S.D.

$$
\begin{aligned}
& \left(\frac{2}{\Gamma^{2}}\right) \Phi\left(\mathbf{x}, \mathbf{x}^{\prime}, \omega\right)=r_{D}(\mathbf{x}) \delta\left(\mathbf{x}-\mathbf{x}^{\prime}\right) N^{N}(\mathbf{x}) \\
& \quad+2 r_{D}(\mathbf{x}) r_{D}\left(\mathbf{x}^{\prime}\right)\langle j(j-1)\rangle r_{f} \sum_{i, n, m=1}^{\infty} C_{i n m} \frac{A_{i}{ }^{N} \psi_{n}(\mathbf{x}) \psi_{m}\left(\mathbf{x}^{\prime}\right)\left(a+D B_{n}{ }^{2}\right)}{\left[2 a+D B_{n}{ }^{2}+D B_{m}{ }^{2}\right]\left[\omega^{2}+\left(a+D B_{n}{ }^{2}\right)^{2}\right]}+O(\epsilon)
\end{aligned}
$$

Although this is identical in form with the expression (85) obtained by ignoring delayed neutrons, it is actually different since the parameters $a$ and $D$ are now related according to the critically condition where delayed neutrons are taken into account, i.e.

$$
a+D B_{1}^{2}-\langle\zeta\rangle r_{t}=0
$$

\section{APPENDIX B}

Here we shall consider neutrons in two energy groups, thermal and fast, carrying out calculations of the P.S.D. to lowest order in the small quantity $r_{a} / r_{R} \equiv \epsilon$ (where $r_{R}$ is the removal rate of neutrons from the fast to the thermal group).

The basic equations are generalized for two groups as follows:

$$
\frac{\partial}{\partial t} N^{D}(\mathbf{x}, t)=r_{D}(\mathbf{x}) H(t) N^{N}(\mathbf{x})
$$

where the superscript $N$ refers to the thermal group. The superscript $n$ will refer to the fast group. 
The detector presumably responds to thermal neutrons, so the formula (33) for the P.S.D. is still correct, with the new notation understood.

$$
\left[r_{a}-D \nabla^{2}\right] N^{N}(\mathbf{x})=r_{R} N^{n}(\mathbf{x}),
$$

where $D \equiv r_{a} L^{2}$ is the thermal diffusion coefficient ( $L$ is the diffusion length).

$$
\left[r_{R}-D^{\prime} \Gamma^{2}\right] N^{n}(\mathbf{x})=\langle j\rangle r_{f} N^{*}(\mathbf{x}) \div S(\mathbf{x}),
$$

where $D^{\prime} \equiv r_{R} \tau$ is the fast diffusion coefficient ( $\tau$ is the neutron age). $S(\mathbf{x})$ (the density of external sources) only contributes to the fast group,

$$
\begin{aligned}
& {\left[\frac{\partial}{\partial t}+r_{a}-D \nabla^{2}\right] \mathbf{X}^{N D}\left(\mathbf{x}, \mathbf{x}^{\prime}, t\right)=r_{R} \mathbf{X}^{n D}\left(\mathbf{x}, \mathbf{x}^{\prime}, t\right)+r_{D}\left(\mathbf{x}^{\prime}\right) H(t) \mathbf{X}^{N N}\left(\mathbf{x}, \mathbf{x}^{\prime}\right)} \\
& -r_{D}\left(\mathbf{x}^{\prime}\right) H(t) \delta\left(\mathbf{x}-\mathbf{x}^{\prime}\right) N^{N}, \\
& {\left[\frac{\partial}{\partial t}+r_{R}-D^{\prime} \nabla^{2}\right] \mathrm{X}^{n D}\left(\mathbf{x}, \mathbf{x}^{\prime}, t\right)=\langle j\rangle r_{f} X^{N D}\left(\mathbf{x}, \mathbf{x}^{\prime}, t\right) \div r_{D}\left(\mathbf{x}^{\prime}\right) H(t) \mathbf{X}^{n x}\left(\mathbf{x}, \mathbf{x}^{\prime}\right),} \\
& {\left[2 r_{12}-D \Gamma^{2}-D \nabla^{\prime 2}\right] \mathrm{X}^{N N}\left(\mathbf{x}, \mathbf{x}^{\prime}\right)=r_{R} \mathrm{X}^{n v}\left(\mathbf{x}, \mathbf{x}^{\prime}\right) \div r^{r} \mathrm{X}^{n N^{\prime}}\left(\mathbf{x}^{\prime}, \mathbf{x}\right)} \\
& +\delta\left(\mathbf{x}-\mathbf{x}^{\prime}\right)\left[r_{a} N^{N}(\mathbf{x})+r_{R} N^{n}(\mathbf{x})\right] \\
& +2 D \nabla \cdot \nabla^{\prime} \delta\left(\mathbf{x}-\mathrm{x}^{\prime}\right) N^{N}(\mathrm{x}) \text {, } \\
& {\left[r_{a}+r_{R}-D \Gamma^{2}-D^{\prime} \nabla^{\prime 2}\right] \mathrm{X}^{n N}\left(\mathbf{x}^{\prime}, \mathbf{x}\right)=r_{R} \mathrm{X}^{n n}\left(\mathbf{x}, \mathbf{x}^{\prime}\right)+\langle j\rangle r_{f} \mathrm{X}^{N N}\left(\mathbf{x}, \mathbf{x}^{\prime}\right)} \\
& -\delta\left(\mathbf{x}-\mathbf{x}^{\prime}\right)\left[r_{R} N^{n}(\mathbf{x}) \div\langle j\rangle r_{f} N^{N}(\mathbf{x})\right], \\
& {\left[2 r_{R}-D^{\prime} \Gamma^{2}-D^{\prime} \nabla^{\prime 2}\right] \mathrm{X}^{n n}\left(\mathbf{x}, \mathbf{x}^{\prime}\right)=\langle j\rangle r_{f} \mathrm{X}^{n_{N} v}\left(\mathbf{x}, \mathbf{x}^{\prime}\right)+\langle j\rangle r_{f} \mathrm{X}^{n-1}\left(\mathbf{x}^{\prime}, \mathbf{x}\right)} \\
& +\delta\left(\mathbf{x}-\mathbf{x}^{\prime}\right)\left[S(\mathbf{x})+r_{R} N^{n}(\mathbf{x})+\left\langle j^{2}\right\rangle r_{f} N^{N}(\mathbf{x})\right] \\
& +2 D^{\prime} \boldsymbol{\nabla} \cdot \boldsymbol{\nabla} \delta\left(\mathbf{x}-\mathbf{x}^{\prime}\right) N^{n}(\mathbf{x}) \text {, } \\
& \frac{\partial}{\partial t} \mathrm{X}^{D D}\left(\mathbf{x}, \mathbf{x}^{\prime}, t\right)=r_{D}(\mathbf{x}) H(t) \mathrm{X}^{N D}\left(\mathbf{x}, \mathbf{x}^{\prime}, t\right) \div r_{D}\left(\mathbf{x}^{\prime}\right) H(t) \mathrm{X}^{N D}\left(\mathbf{x}^{\prime}, \mathbf{x}, t\right) \\
& +r_{D}(\mathbf{x}) H(t) \delta\left(\mathbf{x}-\mathbf{x}^{\prime}\right) N^{N}(\mathbf{x}) \text {. }
\end{aligned}
$$

For simplicity, we have considered the reactor to be in the steady state, no delayed neutron production, and no fission or capture in the fast group. In addition the approximation scheme discussed in Section 3 has been applied.

As in Appendix A, an eigenfunction expansion leads to the following set of coupled equations for the expansion coefficients of $\mathrm{X}^{N N}\left(\mathbf{x}, \mathbf{x}^{\prime}\right), \mathrm{X}^{n N}\left(\mathbf{x}, \mathbf{x}^{\prime}\right), \mathrm{X}^{n N}\left(\mathbf{x}^{\prime}, \mathbf{x}\right)$ and $\mathrm{X}^{n n}\left(\mathbf{x}, \mathbf{x}^{\prime}\right)$ : (extrapolated boundaries are assumed the same for the fast and thermal group).

$$
\begin{gathered}
{\left[2 r_{a}+D B_{n}{ }^{2}+D B_{m}{ }^{2}\right] A_{n m}{ }^{N N}-r_{R} A_{n m}{ }^{n N}-r_{R} A_{m n}{ }^{n N}=\sum_{i=1}^{\infty} C_{i n m} A_{i}{ }^{N}\left(2 r_{a}+D B_{n}{ }^{2}+D B_{m}{ }^{2}\right),} \\
-\langle j\rangle r_{f} A_{n m}{ }^{N N}+\left[r_{a}+r_{n}+D B_{n}{ }^{2}+D^{\prime} B_{m}{ }^{2}\right] A_{n m}{ }^{n N}-r_{R} A_{n m}{ }^{n n}=-\sum_{i=1}^{\infty} C_{i n m}\left[r_{R} A_{i}{ }^{n}+\langle j\rangle r_{f} A_{i}{ }^{N}\right], \\
-\langle j\rangle r_{f} A_{n m}{ }^{N N}+\left[r_{a}+r_{R}+D B_{m}{ }^{2}+D^{\prime} B_{n}{ }^{2}\right] A_{m n}{ }^{n N}-r_{R} A_{n m}{ }^{n n}=-\sum_{i=1}^{\infty} C_{i n m}\left[r_{R} A_{i}{ }^{n}+\langle j\rangle r_{f} A_{i}{ }^{N}\right], \\
\text { (B.1 } \\
\cdots\langle j\rangle r_{f} A_{n m}{ }^{n N}-\langle j\rangle r_{f} A_{m n}{ }^{n N}+\left[2 r_{R}+D^{\prime} B_{n}{ }^{2}+D^{\prime} B_{m}{ }^{2}\right] A_{n m}{ }^{n n}=\sum_{i=1}^{\infty} C_{i n m}\left[\langle j(j-1)\rangle r_{f} A_{i}{ }^{N}\right. \\
\left.+\left(2 r_{R}+D^{\prime} B_{n}{ }^{2}+D^{\prime} B_{m}{ }^{2}\right) A_{i}{ }^{n}\right] . \quad \text { (B.1 }
\end{gathered}
$$

The formal solution for $A_{n m}{ }^{N N}$ is :

$$
\begin{aligned}
A_{n m}{ }^{N N}= & \sum_{i=1} C_{i n m}\left\{\left[2 r_{a}+D B_{n}{ }^{2}+D B_{m}{ }^{2}\right] A_{i}{ }^{N} \Delta A+\left[r_{R} A_{i}{ }^{n}+\langle j\rangle r_{f} A_{i}{ }^{N}\right](\Delta B-\Delta C)\right. \\
& \left.-\left[\langle j(j-1)\rangle r_{f} A_{i}{ }^{N}+\left(2 r_{R}+D^{\prime} B_{n}{ }^{2}+D^{\prime} B_{m}{ }^{2}\right) A_{i}{ }^{n}\right] \Delta D\right\} / \Delta M,
\end{aligned}
$$


where

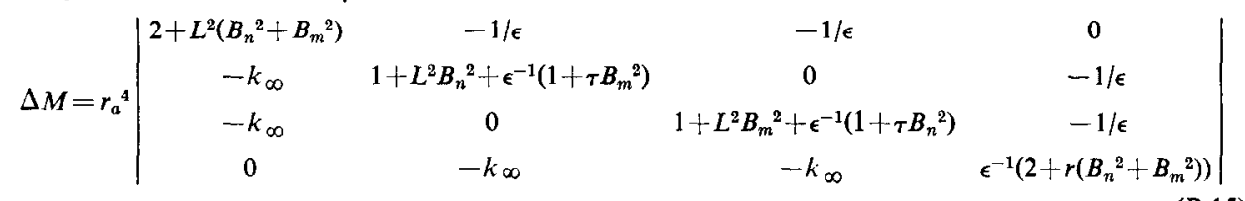$$
=r_{a}{ }^{3}\left[\left(2 r_{a}+D B_{n}{ }^{2}+D B_{m}{ }^{2}\right) \Delta A-\langle j\rangle r_{f}(\Delta B-\Delta C)\right],
$$

and $\Delta A, \Delta B, \Delta C$ and $\Delta D$ are the minors of the elements of the first column of the determinant appearing in $\Delta M$. $k_{\infty}$ is the infinite medium multiplication constant, $\langle i\rangle r_{f} / r_{a}$, and $\epsilon$ is defined as the ratio $r_{a} / r_{R}$. The solution for $A_{n m}{ }^{n N}$ is obtained in a similar fashion:

$$
\begin{aligned}
A_{n m^{n N}}{ }^{n}= & \sum_{i} C_{i n m} r_{a}{ }^{3}\left\{-\left[2 r_{a}+D B_{n}{ }^{2}+D B_{m}{ }^{2}\right] A_{i}{ }^{N} \Delta E-\left[r_{R} A_{i}{ }^{n}+\langle j\rangle r_{f} A_{i}{ }^{N}\right](\Delta F-\Delta G)\right. \\
& \left.+\left[\langle j(j-1)\rangle r_{f} A_{i}{ }^{N}+\left(2 r_{R}+D^{\prime} B_{n}{ }^{2}+D^{\prime} B_{m}{ }^{2}\right) A_{i}{ }^{n}\right] \Delta H\right\} / \Delta M,
\end{aligned}
$$

where $\Delta E, \Delta F, \Delta G$ and $\Delta H$ are the minors of the elements of the second column of $\Delta M$.

We should note that $\Delta M(B .15)$ has the same form as its counterpart in Appendix A. One can obtain therefore, an expression for the P.S.D. to zero-th order in $\epsilon$, just as in Appendix A. It is not necessary to treat the $n=m=1$ term separately.

First we note that to zero-th order in $\epsilon$

$$
\begin{aligned}
& \frac{\Delta A}{\Delta M} \cong \frac{\left(1+\tau B_{n}^{2}\right)\left(1+\tau B_{m}^{2}\right)}{\left(2 r_{a}+r_{u} L^{2}\left(B_{n}{ }^{2}+B_{m}{ }^{2}\right)\right)\left(1+\tau B_{n}^{2}\right)\left(1+\tau B_{m}{ }^{2}\right)-\langle j\rangle r_{f}\left(2+\tau\left(B_{n}{ }^{2}+B_{m}{ }^{2}\right)\right)} \\
& \frac{\Delta B}{\Delta M} \cong-\frac{\Delta A}{\Delta M} \cdot \frac{1}{\left(1+\tau B_{m}^{2}\right)}, \\
& \frac{\Delta C}{\Delta M} \cong \frac{\Delta A}{\Delta M} \cdot \frac{1}{\left(1+\tau B_{n}^{2}\right)} \\
& \frac{\Delta D}{\Delta M} \cong-\frac{\Delta A}{\Delta M} \frac{1}{\left(1+\tau B_{n}^{2}\right)\left(1+\tau B_{m}^{2}\right)}
\end{aligned}
$$

It thus follows from equation (B.14) that to lowest order in $\epsilon$

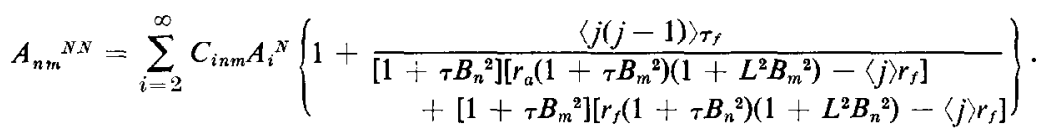

Next we find that to lowest order in $\epsilon$

$$
\frac{\Delta E}{\Delta M} \simeq \frac{\Delta F}{\Delta M} \simeq \frac{\Delta G}{\Delta M} \simeq \frac{\Delta H}{\Delta M} \simeq 0
$$

Thus $A_{n m}{ }^{n v}$ must also vanish to this order. Finally, to obtain the P.S.D. we examine the equation for $A_{n}{ }^{N D}\left(\mathbf{x}^{\prime}, t\right)$ which follows directly from (B.4) and (B.5):

$$
\begin{gathered}
{\left[\frac{\epsilon}{r_{a}} \frac{\partial^{2}}{\partial t^{2}}+\left(1+\tau B_{n}^{2}\right) \frac{\partial}{\partial t} \mid \epsilon\left(1+L^{2} B_{n}{ }^{2}\right) \frac{\partial}{\partial t}+\left(r_{a}\left(1+\tau B_{n}^{2}\right)\left(1+L^{2} B_{n}{ }^{2}\right)-\langle j\rangle r_{f}\right)\right] A_{n}{ }^{N D}\left(\mathbf{x}^{\prime}, t\right)} \\
=r_{D}\left(\mathbf{x}^{\prime}\right) H(t)\left(1+\tau B_{n}^{2}\right) \sum_{m=1}^{\infty}\left[A_{n m}{ }^{N N}-\sum_{i=1}^{\infty} C_{i n m} A_{i}{ }^{N}\right] \psi_{m}\left(\mathbf{x}^{\prime}\right)+r_{D}\left(\mathbf{x}^{\prime}\right) H(t) \sum_{m=1}^{\infty} A_{n m}{ }^{n N} \psi_{m}\left(\mathbf{x}^{\prime}\right) \\
+r_{D}\left(\mathbf{x}^{\prime}\right) \epsilon \frac{\delta(t)}{r_{u}} \sum_{m=1}^{\infty}\left[A_{n m}{ }^{N N}-\sum_{i=1}^{\infty} C_{i n m} A_{i}{ }^{N}\right] \psi_{m}\left(\mathbf{x}^{\prime}\right) .
\end{gathered}
$$


The bracket in the first term on the r.h.s. is considerably simplified by the use of (B.21). Using the approximations of equation (50) we solve (B.23) to lowest order in $\epsilon$, and insert the result into the definition (33) for the generalized P.S.D. The final result is:

$$
\begin{aligned}
& \left(\frac{2}{\Gamma^{2}}\right) \Phi\left(\mathbf{x}, \mathbf{x}^{\prime}, \omega\right)=r_{D}(\mathbf{x}) \delta\left(\mathbf{x}-\mathbf{x}^{\prime}\right) N^{N}(\mathbf{x}) \\
& +r_{D}(\mathbf{x}) r_{D}\left(\mathbf{x}^{\prime}\right) \sum_{i, n, m=1}^{\infty} \frac{\langle j(j-1)\rangle r_{f} C_{i n m} \psi_{n}(\mathbf{x}) \psi_{m}\left(\mathbf{x}^{\prime}\right)\left(a+D_{n} B_{n}{ }^{2}\right) A_{i}{ }^{N}}{\left(1+\tau B_{n}{ }^{2}\right)\left(1+\tau B_{m}{ }^{2}\right)\left[2 a+D_{n} B_{n}{ }^{2}+D_{m} B_{m}{ }^{2}\right]\left[\left(a+D_{n} B_{n}{ }^{2}+\omega^{2}\right]\right.} \\
& \quad+r_{D}(\mathbf{x}) r_{D}\left(\mathbf{x}^{\prime}\right) \sum_{i, n, m=1}^{\infty} \frac{\langle j(j-1)\rangle r_{f} C_{i n m} \psi_{n}\left(\mathbf{x}^{\prime}\right) \psi_{m}(\mathbf{x})\left(a+D_{m} B_{m}{ }^{2}\right) A_{i}{ }^{N}}{\left(1+\tau B_{n}{ }^{2}\right)\left(1+\tau B_{m}{ }^{2}\right)\left[2 a+D_{n} B_{n}{ }^{2}+D_{m} B_{m}{ }^{2}\right]\left[\left(a+D_{m} B_{m}{ }^{2}\right)^{2}-\omega^{2}\right]},
\end{aligned}
$$

where $D_{n}$ is defined according to

$$
D_{n}=r_{a}\left[L^{2} \div \frac{k_{\infty}}{1-\tau B_{n}^{2}} \tau\right] \text {. }
$$

This is just equation (85) with extra factors of $\left[\left(1+\tau B_{n}{ }^{2}\right)\left(1+\tau B_{m}{ }^{2}\right)\right]^{-1}$ and with an 'effective' migration length $M_{n}^{2} \equiv L^{2}+k_{\infty} \tau /\left(1+\tau B_{n}^{2}\right)$ for the $n$-th mode. For the higher modes $M_{n}{ }^{2} \approx L^{2}$, but the extra factors become small and supress the contribution of these terms.

For the $n=m=1$ mode, since

$$
\frac{k_{\infty}}{1+\tau B_{1}{ }^{2}} \approx 1, \quad \frac{1}{1+\tau B_{1}{ }^{2}} \approx 1
$$

the result is the same as the lowest mode result of the one-group treatment, with $L^{2}$ replaced by the migration length $M^{2} \equiv L^{2}+\tau$. It should be pointed out that

where

$$
a+D_{1} B_{1}=(1-k) / /^{\mathrm{h}}
$$

$$
k \equiv\langle j\rangle r_{f} / r_{a}\left(1+\tau{ }^{2}{ }_{1}^{2}\right)\left(1+L^{2} B_{1}{ }^{2}\right), \text { and } l^{\text {th }} \equiv 1 / r_{a}\left(1+L^{2} B_{1}{ }^{2}\right) \text {. }
$$

Thus one obtains the familiar form $\sim\left(\omega^{2}+\left(1-k / l^{\text {th }}\right)^{2}\right)^{-1}$ for the frequency dependence of the P.S.D. The thermal lifetime $l^{\text {th }}$ occurs here rather than the expected total lifetime. Of course

$$
l^{\text {roT }}=l^{\text {th }}(1+O(\epsilon))
$$

and the occurrence of $l^{\text {th }}$ rather than $l^{\text {TOT }}$ in our result is due to the neglect of terms of order $\epsilon$.

\section{APPENDIX $C$}

Here we apply the two energy group model of Appendix B to the infinite homogenous reactor. We shall show that in the frequency range of experimental interest $a \approx \omega<r_{a}$ and to lowest order in the small quantity $r_{a} / r_{R}$ the P.S.D. results of Section 3 are modified merely by the replacement of $L^{2}$ by $M^{2}$ in the diffusion coefficient.

The two-group equations of Appendix B in the infinite reactor limit become:

$$
\begin{aligned}
& \frac{\partial}{\partial t} N^{D}(\mathbf{x}, t)=r^{D}(\mathbf{x}) H(t) N^{N} . \\
& r_{a} N^{N}=r_{R} N^{n} \text {. } \\
& r_{R} N^{n}=\langle j\rangle r_{f} N^{N}+S \text {. } \\
& {\left[\frac{\partial}{\partial t}+r_{a}-D \nabla^{2}\right] \mathrm{X}^{N D}\left(\mathbf{x}, \mathbf{x}^{\prime}, t\right)=r_{R} \mathrm{X}^{n D}\left(\mathbf{x}, \mathbf{x}^{\prime}, t\right)+r_{D}\left(\mathbf{x}^{\prime}\right) H(t) \mathrm{X}^{N N}\left(\mathbf{x}, \mathbf{x}^{\prime}\right)} \\
& -r_{D}\left(\mathbf{x}^{\prime}\right) H(t) \delta\left(\mathbf{x}-\mathbf{x}^{\prime}\right) N^{N} \\
& {\left[\frac{\partial}{\partial t}-r_{R}-D^{\prime} \nabla^{2}\right] \mathrm{X}^{n D}\left(\mathbf{x}, \mathbf{x}^{\prime}, t\right)=\langle j\rangle r_{f} \mathrm{X}^{N D}\left(\mathbf{x}, \mathbf{x}^{\prime}, t\right) \div r_{D}\left(\mathbf{x}^{\prime}\right) H(t) \mathrm{X}^{n N}\left(\mathbf{x}, \mathbf{x}^{\prime}\right) \text {. }} \\
& {\left[r_{a}-D \nabla^{\prime \prime 2}\right] \mathrm{X}^{N N}\left(\mathbf{x}^{\prime \prime}\right)=r_{R} \mathbf{X}^{n N}\left(\mathbf{x}^{\prime \prime}\right)+\delta\left(\mathbf{x}^{\prime \prime}\right) r_{a} N^{N}-D \nabla^{\prime \prime 2} \delta\left(\mathbf{x}^{\prime \prime}\right) N^{N},} \\
& {\left[r_{a}+r_{R}-\left(D+D^{\prime}\right) \nabla^{\prime \prime}\right] \mathrm{X}^{n N}\left(\mathbf{x}^{\prime \prime}\right)=r_{R} \mathrm{X}^{n n}\left(\mathbf{x}^{\prime \prime}\right)+\langle j\rangle r_{f} \mathrm{X}^{N N}\left(\mathbf{x}^{\prime \prime}\right)-\delta(\mathbf{x})\left[r_{R} N^{n}+\langle j\rangle r_{f} N^{N}\right] .} \\
& {\left[r_{R}-D^{\prime} \nabla^{\prime \prime}\right] \mathrm{X}^{n n}\left(\mathbf{x}^{\prime \prime}\right)=\langle j\rangle r_{f} \mathbf{X}^{n N}\left(\mathbf{x}^{\prime \prime}\right)+\delta\left(\mathbf{x}^{\prime \prime}\right)\left[r_{R} N^{n}+\frac{\langle j(j-1)\rangle}{2} r_{f} N^{N}\right]-D^{\prime} \nabla^{\prime \prime 2} \delta\left(\mathbf{x}^{\prime \prime}\right) N^{n}} \\
& \frac{\partial}{\partial t} \mathrm{X}^{D D}\left(\mathbf{x}, \mathbf{x}^{\prime}, t\right)=r_{D}(\mathbf{x}) H(t) \mathrm{X}^{N D}\left(\mathbf{x}, \mathbf{x}^{\prime}, t\right)+r_{D}\left(\mathbf{x}^{\prime}\right) H(t) \mathrm{X}^{N D}\left(\mathbf{x}^{\prime}, \mathbf{x}, t\right)+r_{D}(\mathbf{x}) H(t) \delta\left(\mathbf{x}-\mathbf{x}^{\prime}\right) N^{N} \text {. }
\end{aligned}
$$


If we define

and

$$
G^{N N}\left(\mathbf{x}^{\prime \prime}\right) \equiv \mathrm{X}^{N N}\left(\mathbf{x}^{\prime \prime}\right)-\delta\left(\mathbf{x}^{\prime \prime}\right) N^{N}
$$

$$
G^{n n}\left(\mathbf{x}^{\prime \prime}\right) \equiv \mathbf{X}^{n n}\left(\mathbf{x}^{\prime \prime}\right)-\delta\left(\mathbf{x}^{\prime \prime}\right) N^{N},
$$

then equations (C.6)-(C.8) become:

$$
\begin{gathered}
{\left[r_{a}-D \nabla^{\prime \prime 2}\right] G^{N N}\left(\mathbf{x}^{\prime \prime}\right)=r_{R} \mathrm{X}^{n N}\left(\mathbf{x}^{\prime \prime}\right),} \\
{\left[r_{a}+r_{R}-\left(D+D^{\prime}\right) \nabla^{\prime \prime}\right] \mathrm{X}^{n N}\left(\mathbf{x}^{\prime \prime}\right)=r_{R} G^{n n}\left(\mathbf{x}^{\prime \prime}\right)+\langle j\rangle r_{f} G^{N N}\left(\mathbf{x}^{\prime \prime}\right),} \\
{\left[r_{R}-D^{\prime} \nabla^{\prime \prime 2}\right] G^{n n}\left(\mathbf{x}^{\prime \prime}\right)=\langle j\rangle r_{f} \mathrm{X}^{n N}\left(\mathbf{x}^{\prime \prime}\right)+\delta\left(\mathbf{x}^{\prime \prime}\right) \frac{\langle j(j-1)\rangle}{2} r_{f} N^{N} .}
\end{gathered}
$$

The coupled set of equations (C.11)-(C.13) may be solved for $G^{N N}\left(\mathbf{x}^{\prime \prime}\right)$ and $\mathrm{X}^{n N}\left(\mathbf{x}^{\prime \prime}\right)$ by Fourier transform.

$$
G^{N N}\left(\mathbf{x}^{\prime \prime}\right)=\frac{r_{R}^{2}\langle j(j-1)\rangle r_{f} N^{N}}{2(2 \pi)^{3}} \int \mathrm{d}^{3} \mathbf{k} \frac{\exp i \mathbf{k} \cdot \mathbf{x}^{\prime \prime}}{\left(r_{a}+r_{R}+\left(D+D^{\prime}\right) k^{2}\right)\left[\left(r_{a}+D k^{2}\right)\left(r_{R}+D^{\prime} k^{2}\right)-\langle j\rangle r_{f} r_{R}\right]}
$$

and

$$
\mathrm{X}^{n N}\left(\mathbf{x}^{\prime \prime}\right)=\frac{r_{R}\langle j(j-1)\rangle r_{f} N^{N}}{2(2 \pi)^{3}} \int \mathrm{d}^{3} \mathbf{k} \frac{\exp \left(-i \mathbf{k} \cdot \mathbf{x}^{\prime \prime}\right)\left[r_{a}+D k^{2}\right\rfloor}{\left(r_{a}+r_{R}+\left(D+D^{\prime}\right) k^{2}\right)\left[\left(r_{a}+D k^{2}\right)\left(r_{R}+D^{\prime} k^{2}\right)-\langle j\rangle r_{f} r_{R}\right]}
$$

Next equations (C.4) and (C.5) are Laplace transformed and combined to give the following relationship between $\mathrm{X}^{N D}\left(\mathbf{x}, \mathbf{x}^{\prime}, s\right), G^{N N}\left(\mathbf{x}^{\prime \prime}\right)$ and $\mathrm{X}^{n N}\left(\mathbf{x}^{\prime \prime}\right)$.

$$
\begin{aligned}
{\left[S^{2}+\left(r_{a}+r_{R}-\left(D+D^{\prime}\right) \nabla^{2}\right) S+\left(r_{a}-D \nabla^{2}\right)\left(r_{R}-D^{\prime} \nabla^{2}\right)-\langle j\rangle r_{f} r^{R}\right] \mathrm{X}^{N D}\left(\mathbf{x}, \mathbf{x}^{\prime}, s\right) } \\
=\frac{r_{D}\left(\mathbf{x}^{\prime}\right)}{S}\left[r_{R}-D^{\prime} \nabla^{2}+s\right] G^{N v}\left(\mathbf{x}, \mathbf{x}^{\prime}\right)+\frac{r_{D}\left(\mathbf{x}^{\prime}\right)}{S} r_{R} \mathrm{X}^{n v}\left(\mathbf{x}, \mathbf{x}^{\prime}\right)
\end{aligned}
$$

Solving (C.16) one obtains

$S r_{D}(\mathbf{x}) \mathbf{X}^{N D}\left(\mathbf{x}, \mathbf{x}^{\prime}, s\right)=r_{D}(\mathbf{x}) r_{D}\left(\mathbf{x}^{\prime}\right) \frac{\langle j(j-1)\rangle r_{f} r_{R}{ }^{2} N^{N}}{2(2 \pi)^{3}} \int \mathrm{d}^{3} \mathbf{k} \frac{\exp \left(-i \mathbf{k} \cdot\left(\mathbf{x}-\mathbf{x}^{\prime}\right)\right)\left[S+y_{2}(k)\right]}{\left[S^{2}+S y_{1}(k)+y_{2}(k)\right] y_{1}(k) y_{2}(k)}$, where

and

$$
\begin{aligned}
& y_{1}(k)=r_{a}+r_{R}+\left(D+D^{\prime}\right) k^{2}, \\
& y_{2}(k)=\left(r_{a}+D k^{2}\right)\left(r_{R}+D^{\prime} k^{2}\right)-\langle j\rangle r_{r} r_{R} .
\end{aligned}
$$

Thus using the definition of the infinite medium generalized P.S.D. (33) we can write

$$
\begin{gathered}
\left(\frac{2}{\Gamma^{2}}\right) \Phi\left(\mathbf{x}, \mathbf{x}^{\prime}, \omega\right)=r_{D}(\mathbf{x}) \delta\left(\mathbf{x}-\mathbf{x}^{\prime}\right) N^{N}+r_{D}(\mathbf{x}) r_{D}\left(\mathbf{x}^{\prime}\right) \frac{\langle j(j-1)\rangle r_{f} r_{R}^{2} N^{N}}{(2 \pi)^{3}} \\
\int \mathrm{d}^{3} \mathbf{k} \frac{\exp \left(-i \mathbf{k} \cdot\left(\mathbf{x}-\mathbf{x}^{\prime}\right)\right)}{\left[y_{2}(k)-\omega^{2}+i \omega y_{1}(k)\right]\left[\gamma_{2}(k)-\omega^{2}-i \omega y_{1}(k)\right]} .
\end{gathered}
$$

The integral in (C.20) can be evaluated by the residue theorem.

$\frac{1}{(2 \pi)^{3}} \int \mathrm{d}^{3} \mathbf{k} \frac{\exp \left(-i \mathbf{k} \cdot\left(\mathbf{x}-\mathbf{x}^{\prime}\right)\right)}{\left[y_{2}(k)-\omega^{2}+i \omega y_{1}(k)\right]\left[y_{2}(k)-\omega^{2}-i \omega y_{1}(k)\right]}=\frac{1}{\left|\mathbf{x}-\mathbf{x}^{\prime}\right| 4 \pi D D^{\prime}}\left[A+A^{4}-B-B^{4}\right]$,

where

$$
\begin{aligned}
A & \equiv \frac{\exp \left(-\alpha_{1}\left|\mathbf{x}-\mathbf{x}^{\prime}\right|\right)}{\left[y_{2}\left(i \alpha_{1}\right)-\omega^{2}+i \omega y_{1}\left(i \alpha_{1}\right)\right]\left[\alpha_{2}^{2}-\alpha_{1}^{2}\right]}, \\
B & \equiv \frac{\operatorname{cxp}\left(-\alpha_{2}\left|\mathbf{x}-\mathbf{x}^{\prime}\right|\right)}{\left[y_{2}\left(i \alpha_{2}\right)-\omega^{2}+i \omega y_{1}\left(i \alpha_{2}\right)\right]\left[\alpha_{2}{ }^{2}-\alpha_{1}^{2}\right]},
\end{aligned}
$$

and $i \alpha_{1}, i \alpha_{1}{ }^{*}, i \alpha_{2}, i \alpha_{2}{ }^{*}$ are the four zeros of the denominator in (C.20) located in the upper half $k$ plane. To lowest order in the small quantities $r_{a} / r_{R}$ and $\omega / r_{R}$

$$
\alpha_{1} \cong \sqrt{\frac{\sqrt{\left(M^{2} / \tau\right)^{2}+\left(\omega / r_{a}\right)^{2}}+\left(M^{2} / \tau\right)}{2 L^{2}}}+i \sqrt{\frac{\sqrt{\left(M^{2} / \tau\right)^{2}+\left(\omega / r_{a}\right)^{2}}-\left(M^{2} / \tau\right)}{2 L^{2}}},
$$


and

$$
\alpha_{2} \cong \sqrt{\frac{\sqrt{a^{2}+\omega^{2}+a}}{2 r_{a} M^{2}}}+i \sqrt{\frac{\sqrt{a^{2}+\omega^{2}}-a}{2 r_{a} M^{2}}} .
$$

Now as the real part of $\alpha_{1}$ is much greater than the real part of $\alpha_{2}$ the contributions from $A$ and $A^{*}$ to $\Phi\left(\mathbf{x}, \mathbf{x}^{\prime}, \omega\right)$ can be ignored. We find, therefore, that to lowest order in $\omega / r_{R}$ and $r_{a} / r_{R}$ the generalized P.S.D. is:

$$
\begin{aligned}
\left(\frac{2}{\Gamma^{2}}\right) & \Phi\left(\mathbf{x}, \mathbf{x}^{\prime}, \omega\right)=r_{D}(\mathbf{x}) \delta\left(\mathbf{x}-\mathbf{x}^{\prime}\right) N^{y}+r_{D}(\mathbf{x}) r_{D}\left(\mathbf{x}^{\prime}\right) \frac{\langle j(j-1)\rangle r_{f}}{4 \pi r_{a} M^{2}}-\exp -\left(\sqrt{\frac{\sqrt{a^{2}+\omega^{2}}-a}{2 r_{a} M^{2}}\left|\mathbf{x}-\mathbf{x}^{\prime}\right|}\right. \\
& \times\left\{\frac{\frac{\omega}{r_{a}}\left(\frac{2 L^{2} \tau}{M^{4}}\right) \cos \sqrt{\frac{\sqrt{a^{2}+\omega^{2}}-a}{2 r_{a} M^{2}}}\left|\mathbf{x}-\mathbf{x}^{\prime}\right|+\sin \sqrt{\frac{\sqrt{a^{2}+\omega^{2}-a^{2}}}{2 r_{a} M^{2}}-\left|\mathbf{x}-\mathbf{x}^{\prime}\right|}}{\omega\left[1+\left(\frac{\omega}{r_{a}}\right)^{2}\left(\frac{2 L^{2} \tau}{M^{2}}\right)^{2}\right]}\right\}
\end{aligned}
$$

From equation (59) the one-group generalized P.S.D. is obtained directly. It is:

$$
\begin{gathered}
\left(\frac{2}{\Gamma^{2}}\right) \Phi\left(\mathbf{x}, \mathbf{x}^{\prime}, \omega\right)=r_{D}(\mathbf{x}) \delta\left(\mathbf{x}-\mathbf{x}^{\prime}\right) N^{N}+r_{D}(\mathbf{x}) r_{D}\left(\mathbf{x}^{\prime}\right) \frac{\langle j(j-1)\rangle r_{f} \exp -\left(\sqrt{\left.\frac{\sqrt{a^{2}+\omega^{2}+a}}{2 r_{a} L^{2}}\left|\mathbf{x}-\mathbf{x}^{\prime}\right|\right)}\right.}{4 \pi r_{a} L^{2}\left|\mathbf{x}-\mathbf{x}^{\prime}\right|} \\
\text { (C.27) } \\
\times\left\{\frac{\sin \sqrt{\frac{\sqrt{a^{2}+\omega^{2}}-a}{2 r_{a} L^{2}}\left|\mathbf{x}-\mathrm{x}^{\prime}\right|}}{\omega}\right\} .
\end{gathered}
$$

In the frequency range of experimental interest $1 \gg\left(\omega / r_{a}\right)\left(2 L^{2} \tau / M^{2}\right)$. Thus it is clear that (C.24) and (C.25) are identical except for the replacement of $L^{2}$ by $M^{2}$ and the presence of the cosine term in (C.24). But it is necessary only to note that the presence of the factor $\omega / r_{a}\left(2 L^{2} \tau / M^{4}\right)$, typically $10^{-3}-$ $10^{-5}$, in front of the cosine term means that the sine term dominates except for a narrow range of values of $\mathbf{x}$ and $\mathbf{x}^{\prime}$. Hence, when the integration over detector volume is carried out it is easy to see that the main contribution to the P.S.D., $\Phi(\omega)$, will come from the sine term, except possibly in the limiting case of small detector size. In this limit the integration over $\mathbf{x}$ and $\mathbf{x}^{\prime}$ may be performed easily by replacing the cosine and the exponential by unity and the sine by its argument. With this approximation we find immediately for a spherical detector of radius $R$ :

$$
\frac{\Phi_{\text {Cosine term }}(\omega)}{\Phi_{\text {Sine term }}(\omega)} \cong \frac{\frac{\omega}{r_{a}}\left(\frac{3 L^{2} \tau}{M^{4}}\right)}{\frac{R}{M} \sqrt{\frac{\sqrt{a^{2}+\omega^{2}}-a}{2 r_{a}}}} .
$$

Even for a detector as small as $1 \mathrm{~cm}$ radius, this ratio does not exceed $10^{-1}$ in the frequency range of interest. 